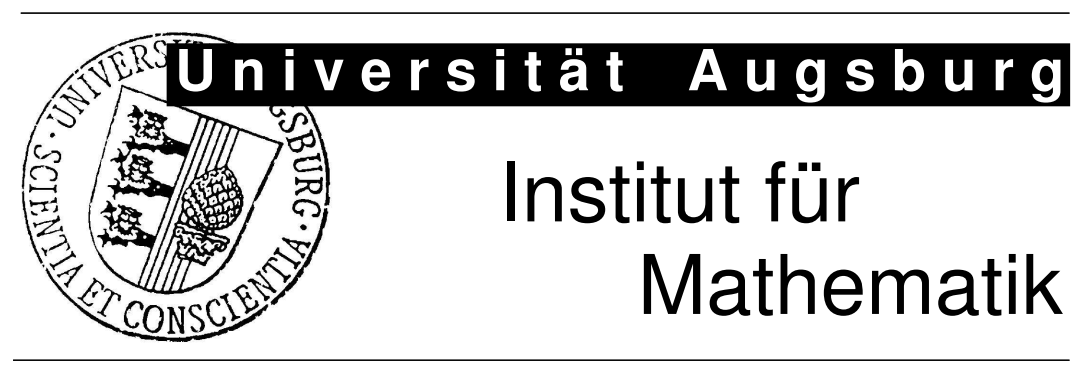

A. Gantner, R. H. W. Hoppe, D. Köster, K. G. Siebert, A. Wixforth Numerical Simulation of Piezoelectrically Agitated Surface Acoustic Waves on Microfluidic Biochips 


\title{
Numerical Simulation of Piezoelectrically Agitated Surface Acoustic Waves on Microfluidic Biochips
}

\author{
Andreas Gantner ${ }^{\star 1}$, Ronald H.W. Hoppe ${ }^{1,2}$, Daniel Köster ${ }^{1}$, Kunibert G. Siebert ${ }^{1}$, Achim \\ Wixforth ${ }^{3}$ \\ 1 Institut für Mathematik, Universität Augsburg, D-86159 Augsburg, Germany \\ 2 Department of Mathematics, University of Houston, Houston, TX 77204-3008, U.S.A. \\ 3 Institut für Physik, Universität Augsburg, D-86159 Augsburg, Germany \\ (http://scicomp.math.uni-augsburg.de; http://www.math.uh.edu)
}

Received: January 00, 2005 / Accepted: January 00, 2005

\begin{abstract}
Microfluidic biochips are biochemical laboratories on the microscale that are used for genotyping and sequencing in genomics, protein profiling in proteomics, and cytometry cell analysis. There are basically two classes of such biochips: active devices, where the solute transport on a network of channels on the chip surface is realized by external forces, and passive chips, where this is done using a specific design of the geometry of the channel network. Among the active biochips, current interest focuses on devices whose operational principle is based on piezoelectrically driven surface acoustic waves generated by interdigital transducers placed on the chip surface.

In this paper, we are concerned with the numerical simulation of such piezoelectrically agitated surface acoustic waves relying on a mathematical model that describes the coupling of the underlying piezoelectric and elastomechanical phenomena. Since the interdigital transducers usually operate at a fixed frequency, we focus on the time-harmonic case. Its variational formulation gives rise to a generalized saddle point problem for which a Fredholm alternative is shown to hold true.

The discretization of time-harmonic surface acoustic wave equations is taken care of by continuous, piecewise polynomial finite elements with respect to a nested hierarchy of simplicial triangulations of the computational domain. The resulting algebraic saddle point problems are solved by block-diagonally preconditioned iterative solvers with preconditioners of BPX-type. Numerical results are given both for a test problem documenting the performance of the iterative solution process and for a realistic surface acoustic wave device illustrating the properties of surface acoustic wave propagation on piezoelectric materials.
\end{abstract}

\footnotetext{
* The first, second and fourth author have been supported by the DFG within the Collaborative Research Center SFB 438. The second author acknowledges further support by the NSF under Grant No. DMS-0411403 and Grant No. DMS0412267
}

\section{Introduction}

Biochips, of the microarray type, are fast becoming the default tool for combinatorial chemical and biological analysis in environmental and medical studies. Programmable biochips are miniaturized biochemical labs that are physically and/or electronically controllable. This technology combines digital photolithography, microfluidics and chemistry. The precise positioning of the samples (e.g., DNA solutes or proteins) on the surface of the chip in pico- to nano-liter volumes can be done either by means of external forces (active devices) or by specific geometric patterns (passive devices).

During the last couple of years, such biochips have attracted a considerable amount of interest, since pharmacology, molecular biology, and clinical diagnostics require the precise handling of precious, tiny samples and costly reagents in amounts of nano-liters. Biochips can transport such volumes and perform biochemical analysis of the samples. Microfluidic biochips and microarrays are used in pharmaceutical, medical and forensic applications as well as in academic research and development for high throughput screening, genotyping and sequencing by hybridization in genomics, protein profiling in proteomics, and cytometry in cell analysis [42]. Traditional technologies rely on fluorescent dyes, radioactive markers, or nano-scale gold-beads based on positive hybridization processes. However, these methods only allow a relatively small number of DNA probes per assay, and they only yield endpoint results and do not provide information about the kinetics of the processes. With the need for better sensitivity, flexibility, cost-effectiveness and a significant speed-up of hybridization, the current technological trend is obtained by the integration of the microfluidics on the chips itself. Very recent and novel devices are surface acoustic wave driven microfluidic biochips whose operational mode is based on piezoelectrically actuated surface acoustic waves on the surface of a chip which transport the droplet containing probe along a lithographically produced network to marker molecules placed at pre-specified surface lo- 


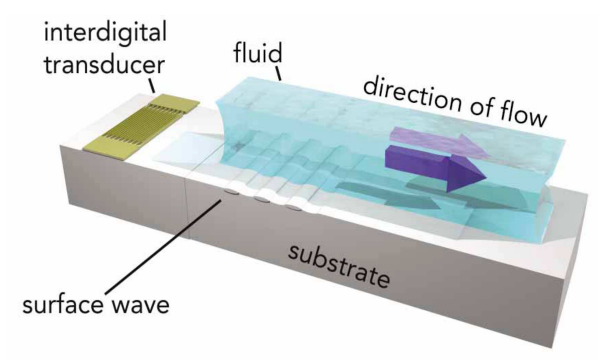

Fig. 1. Working principle of an SAW biochip

cations (cf., e.g., [8,43,54-57]). By changing the surface chemistry appropriately, a fluidic network is produced on the chip: Without mechanical tools the chip is equipped with paths on which samples (and reagents) propagate as if on tracks. This is done lithographically by a lateral modulation of the wetting properties of the surface which leads to pronounced hydrophilic and superhydrophobic regions with significantly different wetting angles. Small amounts of reagents are confined to these tracks in contrast to mechanical barriers used in conventional microfluidics.

The core of the technology are nano-pumps featuring surface acoustic waves generated by electric pulses of high frequency. These waves propagate like a miniaturized earthquake (nano-scale earthquake) and in this way transport liquids along the surface of the chip (cf. Figure 1). Figure 2 below gives an illustration of a nano titration chip. On the fluidic network a small portion of titrate solution (middle) is separated from a larger volume (right). Surface acoustic waves transport this quantity towards the analyte (left) at the reaction site. Once a critical concentration is attained, it can be either detected by a change of the color of the analyte or a change of the conductivity. In the latter case, this can be easily measured by a sensor that is integrated on the same chip. Surface Acoustic Waves (SAW) have been used for

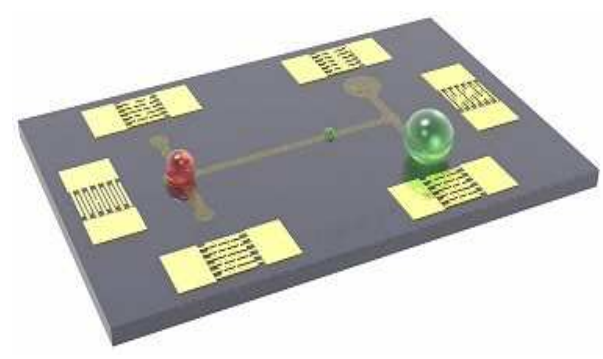

Fig. 2. Fluidic network on the surface of the chip

a long time in high frequency applications (cf., e.g., [13, $36,40]$ and [58]). Using SAW-principles, it is now possible to combine microelectronics and biochemistry. Modern semiconductor technology enables the cost-effective production of devices that unify biological functionality, sensors and pumps for the transport of samples. These devices can be easily integrated in electronic systems like those that are used in point-of-care diagnostics (see [4, $9,45,46,51])$.

The nano-pump consists of a piezoelectric substrate which is equipped with so-called interdigital transducers on the surface. Radio-frequency signals are fed into those transducers and are converted to a deformation of the crystal underground. In this way, a mechanical wave is launched across the surface with wavelengths in the range of a few microns and amplitudes about only a nano-meter. Liquids on the surface are subject to the vibrating force and absorb parts of the energy. The absorption of energy for various frequencies depends on the density and viscosity. These properties are ultimately determined by the atomic composition, molecular structure and dynamics of the fluid.

Surface acoustic waves of larger amplitudes move liquid droplets as a whole whereas low power surface acoustic waves induce some sort of internal streaming. The latter case enables the construction of surface acoustic wave based nano-mixers. If the frequency of the surface acoustic wave is changed, different streaming patterns are induced and superimposed within the droplet that leads to a homogeneous blend of the water and the probe much faster than by more conventional diffusion type microfluidic mixing techniques.

Figure 3 illustrates the effect of nano-mixing in case of the dissolution of a fluorescent dye deposited on the chip surface with agitation (acoustically induced mixing) and without agitation. By using surface acoustic

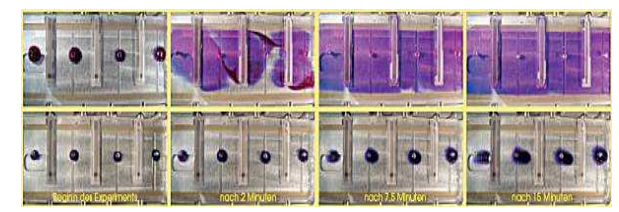

Fig. 3. Surface acoustic wave nano-mixer; with agitation (top), without agitation (below)

wave nano-pumps, different reagents can be efficiently mixed, separated or moved to different reaction sites on the chip. Compared with conventional micro titer plates, the respective volumes are reduced by several orders of magnitudes.

In this article we focus on the excitation of surface acoustic waves by interdigital transducers. The paper is organized as follows: In Section 2, we give a brief outline of the theory of piezoelectricity. In Section 3, we deal with the physical modeling of piezoelectrically driven surface acoustic waves followed by the formulation and analysis of a mathematical solution theory in a variational framework which is provided in Section 4. Section 5 is devoted to the finite element discretization of the time-harmonic surface acoustic wave equations, and Section 6 describes the numerical solution of the resulting algebraic saddle point problem and the associated Schur 
complement system by preconditioned iterative solvers with block-diagonal multilevel preconditioners of BPXtype. Finally, in Section 7 we provide a detailed documentation of numerical results illustrating the performance of the iterative solvers as well as the properties of surface acoustic wave propagation on a realistic piezoelectrical SAW device.

\section{The theory of piezoelectricity}

In piezoelectric materials, the mechanical stress $\boldsymbol{\sigma}$ depends linearly on the electric field $\boldsymbol{E}$, in contrast to nonpiezoelectric materials where the effect is quadratic.

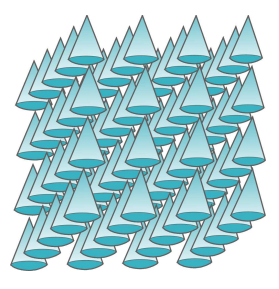

Fig. 4. Polar axis of a piezoelectric crystal

Piezoelectric materials also show the reverse effect to generate an electric field when subjected to mechanical stress. These properties are called the piezoelectric effect and the inverse piezoelectric effect, respectively. The origin of the piezoelectric effect is related to an asymmetry in the unit cell of a piezoelectric crystal and can be observed only in materials with a polar axis, i.e., in face of a rotational symmetry around this axis there are differences in the two directions of this axis (cf. Figure 4). Crystallographers recognize thirty-two classes of crystals of which twenty exhibit the piezoelectric effect. Figure 5 (right) shows a traditional "PZT" piezoelectric material consisting of a small, tetravalent metal ion, usually titanium or zirconium, in a lattice of larger divalent metal ions, usually lead or barium, and $\mathrm{O}_{2}$-ions. Such materials show a simple cubic symmetry above the Curie temperature and are thus isotropic before poling. After poling, they exhibit a tetragonal symmetry below the Curie temperature (see Figure 5 (right). Above this temperature, they lose the piezoelectric properties again.

Although the magnitudes of piezoelectric voltages, movements, or forces are small, and often require amplification (for instance, a typical disc of piezoelectric ceramic will increase or decrease in thickness by only a small fraction of a millimeter), piezoelectric materials have been adapted to a wide range of applications: The piezoelectric effect is used in sensing applications, such as in force or displacement sensors. The inverse piezoelectric effect is used in actuation applications, for instance in motors and devices that precisely control positioning, and in generating sonic and ultrasonic signals. Typical piezoelectric materials are quartz $\left(\mathrm{SiO}_{2}\right)$, lithium niobate $\left(\mathrm{LiNbO}_{3}\right)$ or barium titanate $\left(\mathrm{BaTiO}_{3}\right)$.
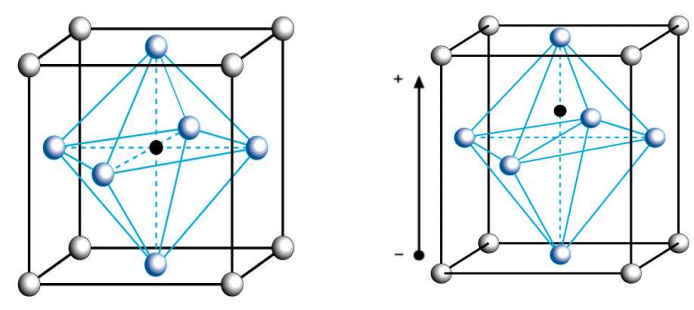

Fig. 5. Crystallographic structure of a "PZT" material: Temperature above (left) and below (right) the Curie point

In the sequel, we consider a linear model for piezoelectricity in which the elastic, piezoelectric and dielectric coefficients are treated as constants independent of the magnitude and frequency of the applied mechanical stresses and electric fields. The model is macroscopic, i.e., only mean values of the relevant physical magnitudes are incorporated. Real materials involve microscopic effects as well as mechanical and electric dissipation and nonlinear behavior. For further reference on piezoelectric problems we refer to $[18,35]$ and the references therein.

Denoting by $\Omega \subset \mathbb{R}^{d}, d=2$ or $d=3$ a Lipschitz domain and by $[0, T] \subset \mathbb{R}_{+}$a time interval, the mechanical displacement $\boldsymbol{u}=\boldsymbol{u}(\boldsymbol{x}, t)$ of a piezoelectric material, occupying $\Omega$ and being exposed to a volume force $\boldsymbol{b}$, is described by the wave equation

$$
\rho \frac{\partial^{2} \boldsymbol{u}}{\partial t^{2}}-\boldsymbol{\nabla} \cdot \boldsymbol{\sigma}=\boldsymbol{b} \quad \text { in } Q:=\Omega \times[0, T] .
$$

Here, $\rho$ is the density and $\boldsymbol{\sigma}=\left(\sigma_{i j}\right)$ stands for stress tensor. The stress tensor is related to the linearized strain tensor $\boldsymbol{\varepsilon}=\frac{1}{2}\left(\boldsymbol{\nabla} \boldsymbol{u}+(\boldsymbol{\nabla} \boldsymbol{u})^{T}\right)$ by the constitutive equation (generalized Hooke's law)

$$
\sigma_{i j}(\boldsymbol{u}, \boldsymbol{E})=c_{i j k l} \varepsilon_{k l}(\boldsymbol{u})-e_{k i j} E_{k} .
$$

Here, $\boldsymbol{E}=\left(E_{k}\right)$ denotes the electric field, $\boldsymbol{c}=\left(c_{i j k l}\right)$ is the symmetric, positive definite forth-order elasticity tensor and $\boldsymbol{e}=\left(e_{k i j}\right)$ refers to the symmetric third-order piezoelectric tensor. Note that here and in the sequel we adopt Einstein's summation convention.

In piezoelectric materials, the frequency of the occurring electric field wave is considered sufficiently small so that the coupling of electromagnetic waves and elastic waves can be neglected. This means that local perturbations in the electromagnetic field are felt almost instantaneously throughout the domain, so that the electric field can be treated as quasi-static. Practically, this can be achieved by setting the magnetic permeability to zero, corresponding to an infinite speed of the electromagnetic wave. Maxwell's second equation then reduces to $\boldsymbol{\nabla} \times \boldsymbol{E}=\mathbf{0}$, i.e., the electric field is irrotational and thus can be represented as the gradient of an electric scalar potential $\Phi$ according to $\boldsymbol{E}=-\boldsymbol{\nabla} \Phi$. When the electric field $\boldsymbol{E}$ is known, the magnetic field $\boldsymbol{H}$ can be obtained from Maxwell's first equation. However, the magnetic field is usually not of interest in piezoelectric computations and is therefore not considered further. Moreover, 
piezoelectric substrates are nearly perfect isolators, i.e., the density of the free electric charges and the current density can be completely neglected. Consequently, the only relevant Maxwell equation is $\boldsymbol{\nabla} \cdot \boldsymbol{D}=0$, where $\boldsymbol{D}=\boldsymbol{D}(\boldsymbol{x}, t)$ is the electric displacement that is related to the electric field $\boldsymbol{E}$ by the constitutive equation

$$
D_{i}=\epsilon_{i j} E_{j}+P_{i} .
$$

Here, $\boldsymbol{P}=\left(P_{i}\right)$ is the electric polarization and $\boldsymbol{\epsilon}=\left(\epsilon_{i j}\right)$ stands for the symmetric, positive definite permittivity tensor.
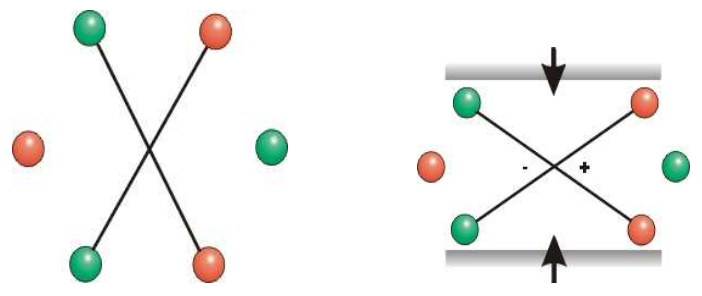

Fig. 6. The formation of an electric dipole by pressure

Figure 6 shows a schematic explanation for the formation of a polarization $\boldsymbol{P}$ in an atomic structure when subjected to external stress: In both cases one can see six "point charges", "red" indicating positive and "green" negative charges. In a relaxed state with no forces acting on them, they are arranged at the vertices of an hexagon. In some distance, the positive and negative array of charges will cancel each other out and the potential $\Phi$ will be zero. If a compressive force is applied to the hexagon, the array is distorted in such a way as to bring two of the positive charges closer together at one end and the negative charges at the other. This forms a dipole where one end of the array is positive and the other one is negative. One can easily imagine a crystal structure made up of these hexagonal arrangements of ions.

In piezoelectric materials, the polarization according to external strain is linear. In analogy to the inverse effect (2), we set

$$
D_{i}(\boldsymbol{u}, \boldsymbol{E})=e_{i k l} \varepsilon_{k l}(\boldsymbol{u})+\epsilon_{i j} E_{j} .
$$

Summarizing, the linear field equations of piezoelectricity are given by

$$
\begin{aligned}
& \rho \frac{\partial^{2} u_{i}}{\partial t^{2}}-c_{i j k l} u_{k, l j}-e_{k i j} \Phi_{, k j}=b_{i} \quad \text { in } Q, \\
& e_{i k l} u_{k, l i}-\epsilon_{i j} \Phi_{, j i}=0 \text { in } Q .
\end{aligned}
$$

and the constitutive equations

$$
\begin{aligned}
\sigma_{i j}(\boldsymbol{u}, \Phi) & =c_{i j k l} \varepsilon_{k l}(\boldsymbol{u})+e_{k i j} \Phi_{, k}, \\
D_{i}(\boldsymbol{u}, \Phi) & =e_{i k l} \varepsilon_{k l}(\boldsymbol{u})-\epsilon_{i j} \Phi_{, j} .
\end{aligned}
$$

The boundary $\partial \Omega$ is partitioned into two disjoint sets according to

$$
\begin{array}{ll}
\partial \Omega=\Gamma_{u} \cup \Gamma_{\sigma}, & \Gamma_{\sigma}=\partial \Omega \backslash \Gamma_{u}, \\
\partial \Omega & =\Gamma_{\Phi} \cup \Gamma_{D}, \quad \Gamma_{D}=\partial \Omega \backslash \Gamma_{\Phi},
\end{array}
$$

where the Dirichlet boundaries $\Gamma_{u}$ and $\Gamma_{\Phi}$ are assumed to be closed and with non-vanishing $(d-1)$-dimensional measure. The piezoelectric equations are supplemented by the decoupled boundary conditions

$$
\begin{aligned}
\left.\boldsymbol{u}\right|_{\Gamma_{u}} & =\boldsymbol{u}_{\Gamma}, \quad \text { where } \partial \Omega=\Gamma_{u} \cup \Gamma_{\sigma}, \\
\left.\boldsymbol{\sigma} \cdot \boldsymbol{n}\right|_{\Gamma_{\sigma}} & =\boldsymbol{\sigma}_{n}, \\
\left.\Phi\right|_{\Gamma_{\Phi}} & =\Phi_{\Gamma}, \quad \text { where } \partial \Omega=\Gamma_{\Phi} \cup \Gamma_{D}, \\
\left.\boldsymbol{D} \cdot \boldsymbol{n}\right|_{\Gamma_{D}} & =D_{n}, \quad \text {, }
\end{aligned}
$$

and by the initial conditions

$$
\boldsymbol{u}(\boldsymbol{x}, 0)=\boldsymbol{u}_{0}(\boldsymbol{x}) \quad, \quad \frac{\partial \boldsymbol{u}}{\partial t}(\boldsymbol{x}, 0)=\boldsymbol{u}_{1}(\boldsymbol{x}) .
$$

Sometimes, it is useful to adopt a compressed notation for the piezoelectric moduli, the Voigt notation (see, e.g., $[18,35,61])$. By utilizing the symmetry properties of the third- and forth-order tensors they can be reduced to higher dimensional second-order matrices. To this end, we use the identification $I=(i j)$, where

\begin{tabular}{|c||c|c|c|c|c|c|}
\hline$(i j)$ & $(11)$ & $(22)$ & $(33)$ & $(23)$ & $(13)$ & $(12)$ \\
\hline$I$ & 1 & 2 & 3 & 4 & 5 & 6 \\
\hline
\end{tabular}

i.e., $c_{I K}=c_{i j k l}, e_{i K}=e_{i k l}$ and $\epsilon_{I}=\epsilon_{i j}$. With this notation, the characteristic properties of a linear piezoelectric substrate are completely determined by the material matrix

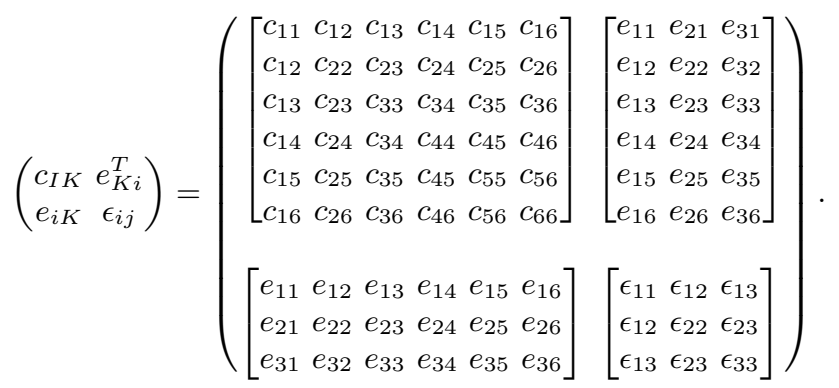

The matrices $c_{I K}$ and $\epsilon_{i j}$ are symmetric with respect to the main diagonal and hence, there are $21+18+6=45$ independent moduli for the most general piezoelectric substrates.

\section{Piezoelectrically driven surface acoustic waves}

\subsection{Surface Acoustic Wave Devices}

Surface acoustic waves (SAWs) are modes of elastic energy propagating at the surface of a solid body. Being the nano-meter size analogon of earthquakes, they have been made available to industrial applications during the last two decades. The underlying technique is relatively new, although the first theoretical treatments on the propagation of surface acoustic waves at the free surface of a homogenous isotropic elastic solid date back approximately 150 years (cf. [44]). But it was not before White and Voltmer [53] succeeded in the production of SAWs on the surface of a piezoelectric substrate that the use of this technology became clear. Nowadays, piezoelectric 
SAW devices are very popular in signal-processing applications (see, e.g., $[13,20,30,36]$ ), which is mostly due to the fact that on homogenous substrates the velocity of SAWs is independent of their frequency.

SAWs are easily excited on piezoelectric solids, because substrates deform due to the application of an electric field. Rapid changes of these electric fields are efficiently converted into a real 'nano-quake on a chip'. Such rapid changes can be generated by a metallic electrode comb structure, called interdigital transducer (IDT), deposited on the surface of the piezoelectric material (see Figure 7 ). By applying an alternating voltage to the IDT,

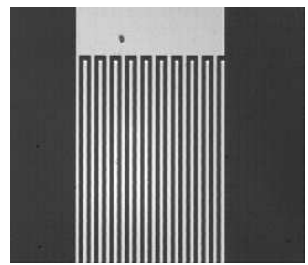

Fig. 7. Interdigital transducer

a surface acoustic wave is excited [39]. Typical frequencies range in the hundred $\mathrm{MHz}$ regime, typical wavelengths of SAW are micrometers. Since the SAW components can be manufactured using advanced photolithographic techniques, they meet the requirements of small size and weight. This is also the reason why SAW devices can be mass produced using the same techniques as in semiconductor microfabrication. They receive outstanding response characteristics, especially in filter applications.

Unfortunately, the excitation of an IDT on a piezoelectric substrate can also lead to the generation of bulk acoustic waves (BAWs) as well as surface waves. In most applications, such BAWs are certainly undesirable, e.g., in signal processing applications they seriously degrade the filter response. In most analytical treatments, BAWs are neglected.

The typical dimensions of an SAW chip are only a few millimeters, depending on the operating frequency. A wide range of piezoelectric materials are used for the production of SAW devices, among them lithium niobate $\left(\mathrm{LiNbO}_{3}\right)$, lithium tantalate $\left(\mathrm{LiTaO}_{3}\right)$, and quartz $\left(\mathrm{SiO}_{2}\right)$ monocrystals.

\subsection{Physical Modeling of Rayleigh Waves}

The type of surface waves considered here are so-called Rayleigh waves (see, e.g., $[3,34]$ ), i.e., these are waves polarized in the sagittal plane and propagating at the free surface at a speed less than that of volume shear elastic waves. These waves usually are considered in a semiinfinite, isotropic and homogenous linear elastic space, which will be fixed as in Figure 8 (left).
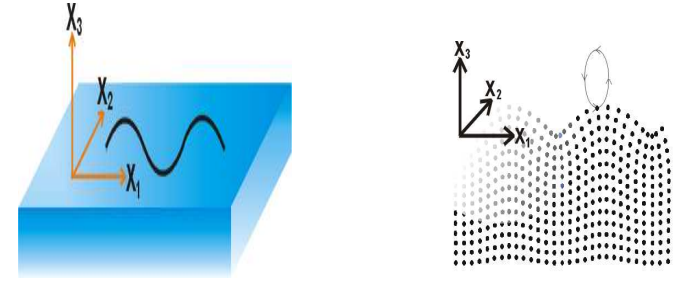

Fig. 8. Modeling of surface acoustic waves. Coordinate system (left) and Rayleigh wave (right)

The sagittal plane is the plane spanned by the real wavevector $\boldsymbol{k}$ and the unit surface normal, i.e., the $\left(x_{1}, x_{3}\right)$ plane in Figure 8 (left). SAWs are strictly confined to the limiting surface of the piezoelectric substrate and practically nil outside a relatively narrow zone. To be precise, the amplitude of the displacement $\boldsymbol{u}$ decays exponentially with depth into the substrate [35]. In true Rayleigh waves, most of the energy $(90 \%)$ is concentrated within one wavelength from the surface. Thus, both the mechanical displacements $\boldsymbol{u}$ and the electric potential $\Phi$ should vanish as $x_{3} \rightarrow \infty$. Moreover, since $x_{1}$ is the direction of propagation of the wave solutions, there is no dependence of $\boldsymbol{u}$ and $\Phi$ on the $x_{2}$ coordinate, since here the surface is assumed to be infinite.

We note that in the physical modeling of surface acoustic waves the assumption of an $x_{2}$-independence is extremely simplifying, since piezoelectric materials are in general anisotropic and pure Rayleigh surface acoustic waves can be observed only in rare crystal cuts.

The two wave motions in the $x_{1}$ - and $x_{3}$-direction are $90^{\circ}$ out of phase in the time domain: if one wave component is at its maximum for a given instant, the other will be zero. Moreover, the displacement in the $x_{3}$-direction will be larger than that in the $x_{1}$-direction. These considerations give rise to

$$
\begin{aligned}
u_{i}\left(x_{1}, x_{3}, t\right) & =\alpha_{i} \exp \left(-\beta k x_{3}\right) \exp \left(\mathrm{i}\left(\omega t-k x_{1}\right)\right), \\
\Phi\left(x_{1}, x_{3}, t\right) & =\alpha_{4} \exp \left(-\beta k x_{3}\right) \exp \left(\mathrm{i}\left(\omega t-k x_{1}\right)\right),
\end{aligned}
$$

with $\operatorname{Re}(\beta)>0$. Hereafter, i denotes the imaginary unit and $k$ the modulus of the wave-vector $\boldsymbol{k}$. In some sense the factor $\beta$ measures the rate of exponential decay into the substrate. We insert these functions into the piezoelectric equations by

$$
\begin{aligned}
\rho \frac{\partial^{2} u_{i}}{\partial t^{2}}-c_{i j k l} \frac{\partial^{2} u_{k}}{\partial x_{l} \partial x_{j}}-e_{k i j} \frac{\partial^{2} \Phi}{\partial x_{k} \partial x_{j}} & =0 \\
e_{i k l} \frac{\partial^{2} u_{k}}{\partial x_{l} \partial x_{i}}-\epsilon_{i j} \frac{\partial^{2} \Phi}{\partial x_{j} \partial x_{i}} & =0 .
\end{aligned}
$$

This leads to a linear system for the coefficients $\alpha_{i}$ of the form

$$
M \boldsymbol{\alpha}=0 .
$$

Here, $\boldsymbol{\alpha}=\left(\alpha_{i}\right) \in \mathbb{C}^{4}, \boldsymbol{M}=\left(M_{k l}\right) \in \mathbb{C}^{4 \times 4}$, and the coefficients $M_{k l}$ are quadratic functions in $\beta$. For the existence of nontrivial solutions, we have to require that 
$\operatorname{det} M=0$. Accounting for $\operatorname{Re}(\beta)>0$, we get 4 possible values for $\beta$. For each such $\beta$, there is an eigenvector $\boldsymbol{\alpha}$.

The general solution is then obtained as a linear combination of these solutions

$$
\begin{aligned}
& u_{i}\left(x_{1}, x_{3}, t\right)=\exp \left(\mathrm{i}\left(\omega t-k x_{1}\right)\right) . \\
& \sum_{m=1}^{4} c^{(m)} \alpha_{i}^{(m)} u_{i}^{0(m)} \exp \left(-\beta^{(m)} k x_{3}\right), \\
& \Phi\left(x_{1}, x_{3}, t\right)=\exp \left(\mathrm{i}\left(\omega t-k x_{1}\right)\right) . \\
& \sum_{m=1}^{4} c^{(m)} \alpha_{4}^{(m)} \Phi^{0(m)} \exp \left(-\beta^{(m)} k x_{3}\right) .
\end{aligned}
$$

We note that the weighting factors $c^{(m)}$, representing the value of the phase velocity, have to be chosen according to the boundary conditions.

The use of anisotropic materials causes many differences in detail even though the occurring surface waves share many features. For instance, the waves are still elliptically polarized at each depth and the displacement amplitude decays exponentially into the substrate. But in anisotropic materials, the phase velocity depends on the direction of propagation and in general, the vector of energy flow is not parallel to the wave vector. Moreover, the plane of the elliptical polarization of the displacement does not necessarily correspond to the sagittal plane, but even when it does, the principal axes of the ellipse are not necessarily $x_{1}$ and $x_{3}$.

\section{Mathematical theory of piezoelectric SAWs}

Surface acoustic waves are usually excited by a source interdigital transducer located at $\Gamma_{\Phi}$ and operating at a fixed frequency $\omega>0$. We treat the excitation as a Dirichlet boundary condition for the electric potential $\Phi$ and assume that there is no further volume force $\boldsymbol{b}$. Consequently, the piezoelectric equations reduce to

$$
\begin{aligned}
\rho \frac{\partial^{2} u_{i}}{\partial t^{2}}-c_{i j k l} \frac{\partial^{2} u_{k}}{\partial x_{l} \partial x_{j}}-e_{k i j} \frac{\partial^{2} \Phi}{\partial x_{k} \partial x_{j}} & =0 \\
e_{i k l} \frac{\partial^{2} u_{k}}{\partial x_{l} \partial x_{i}}-\epsilon_{i j} \frac{\partial^{2} \Phi}{\partial x_{j} \partial x_{i}} & =0 .
\end{aligned}
$$

Hereafter, we use without loss of generality $\rho=1$. We are now looking for time harmonic solutions

$$
\begin{aligned}
& \boldsymbol{u}(\boldsymbol{x}, t)=\operatorname{Re}(\boldsymbol{u}(\boldsymbol{x}) \exp (-\mathrm{i} \omega t)) \\
& \Phi(\boldsymbol{x}, t)=\operatorname{Re}(\Phi(\boldsymbol{x}) \exp (-\mathrm{i} \omega t))
\end{aligned}
$$

where the functions $\boldsymbol{u}$ and $\Phi$ are complex valued. The boundary conditions are assumed to be of the same form

$$
\begin{aligned}
\boldsymbol{u}(\boldsymbol{x}, t) & =\operatorname{Re}\left(\boldsymbol{u}_{\Gamma}(\boldsymbol{x}) \exp (-\mathrm{i} \omega t)\right), \\
\boldsymbol{\sigma}(\boldsymbol{x}, t) \cdot \boldsymbol{n} & =\operatorname{Re}\left(\boldsymbol{\sigma}_{n}(\boldsymbol{x}) \exp (-\mathrm{i} \omega t)\right), \\
\Phi(\boldsymbol{x}, t) & =\operatorname{Re}\left(\Phi_{\Gamma}(\boldsymbol{x}) \exp (-\mathrm{i} \omega t)\right), \\
\left.\boldsymbol{D}(\boldsymbol{x}, t) \cdot \boldsymbol{n}\right|_{\Gamma_{D}} & =\operatorname{Re}\left(D_{n}(\boldsymbol{x}) \exp (-\mathrm{i} \omega t)\right) .
\end{aligned}
$$

For the variational formulation of (11),(12), (15a)-(15d) we adopt standard notation of Lebesgue and Sobolev space theory (cf., e.g., $[1,21,37])$. We denote by $L^{2}(\Omega)$ and $\boldsymbol{L}^{2}(\Omega)$ the Lebesgue of square integrable complex valued functions respectively vector fields on $\Omega$ with inner product $(\cdot, \cdot)_{0, \Omega}$ and norm $\|\cdot\|_{0, \Omega}$. We use $H^{k}(\Omega)$, $\boldsymbol{H}^{k}(\Omega)$ for the Sobolev space of complex valued square integrable functions/vector fields having square integrable weak derivatives up to order $k \in \mathbb{N}$ with inner product $(\cdot, \cdot)_{k, \Omega}$ and norm $\|\cdot\|_{k, \Omega}$. For $\Gamma^{\prime} \subseteq \partial \Omega$, we refer to $H^{\frac{1}{2}}\left(\Gamma^{\prime}\right)$ and $\boldsymbol{H}^{\frac{1}{2}}\left(\Gamma^{\prime}\right)$ as the trace space associated with $H^{1}(\Omega)$ and $\boldsymbol{H}^{1}(\Omega)$. The subspaces $H_{0, \Gamma^{\prime}}^{1}(\Omega), \boldsymbol{H}_{0, \Gamma^{\prime}}^{1}(\Omega)$ stand for the subspaces of functions/vector fields on $\Omega$ with vanishing trace on $\Gamma^{\prime}$ (omitting the subindex $\Gamma^{\prime}$, if $\left.\Gamma^{\prime}=\partial \Omega\right)$. We denote by $H_{00}^{\frac{1}{2}}\left(\Gamma^{\prime}\right) \subset H^{\frac{1}{2}}\left(\Gamma^{\prime}\right)$ and $\boldsymbol{H}_{00}^{\frac{1}{2}}\left(\Gamma^{\prime}\right) \subset \boldsymbol{H}^{\frac{1}{2}}\left(\Gamma^{\prime}\right)$ the subspaces of functions/vector fields whose extension by zero to all of $\partial \Omega$ belongs to $H^{\frac{1}{2}}(\partial \Omega)\left(\boldsymbol{H}^{\frac{1}{2}}(\partial \Omega)\right)$ and defines a bounded linear operator. The associated dual spaces are referred to as $H^{-\frac{1}{2}}\left(\Gamma^{\prime}\right)$ and $\boldsymbol{H}^{-\frac{1}{2}}\left(\Gamma^{\prime}\right)$.

For the ease of notation, we set $\boldsymbol{V}:=\boldsymbol{H}_{0 ; \Gamma_{u}}^{1}(\Omega)$, $W:=H_{0 ; \Gamma_{\Phi}}^{1}(\Omega)$, and we denote by $\boldsymbol{V}^{*}$ and $W^{*}$ the associated dual spaces. As far as the elastic and electric Dirichlet boundary data are concerned, we assume $\boldsymbol{u}_{\Gamma} \in$ $\boldsymbol{H}^{\frac{1}{2}}\left(\Gamma_{u}\right)$ as well as $\Phi_{\Gamma} \in H^{\frac{1}{2}}\left(\Gamma_{\Phi}\right)$. We denote by $\boldsymbol{E}_{\Gamma_{u}}$ : $\boldsymbol{H}^{\frac{1}{2}}\left(\Gamma_{u}\right) \rightarrow \boldsymbol{H}^{1}(\Omega)$ and by $E_{\Gamma_{\Phi}}: H^{\frac{1}{2}}\left(\Gamma_{\Phi}\right) \rightarrow H^{1}(\Omega)$ the harmonic extension operators with $\left.\boldsymbol{E}_{\Gamma_{u}}\left(\boldsymbol{u}_{\Gamma}\right)\right|_{\Gamma_{u}}=\boldsymbol{u}_{\Gamma}$ and $\left.E_{\Gamma_{\Phi}}\left(\Phi_{\Gamma}\right)\right|_{\Gamma_{\Phi}}=\Phi_{\Gamma}$, and we introduce the subspaces

$$
\begin{aligned}
\boldsymbol{V}_{\Gamma_{u}} & :=\boldsymbol{V}+\boldsymbol{E}_{\Gamma_{u}}\left(\boldsymbol{u}_{\Gamma}\right), \\
W_{\Gamma_{\Phi}} & :=W+E_{\Gamma_{\Phi}}\left(\Phi_{\Gamma}\right) .
\end{aligned}
$$

For the elastic and electric Neumann boundary data we suppose that $\boldsymbol{\sigma}_{n} \in \boldsymbol{H}^{-\frac{1}{2}}\left(\Gamma_{\sigma}\right)$ and $D_{n} \in H^{-\frac{1}{2}}\left(\Gamma_{D}\right)$. We refer to $\boldsymbol{R}_{\Gamma_{\sigma}}: \boldsymbol{H}^{1}(\Omega) \rightarrow \boldsymbol{H}^{-\frac{1}{2}}\left(\Gamma_{\sigma}\right)$ and $R_{\Gamma_{D}}: \boldsymbol{H}^{1}(\Omega) \rightarrow$ $\boldsymbol{H}^{-\frac{1}{2}}\left(\Gamma_{D}\right)$ as the trace operators with $\boldsymbol{R}_{\Gamma_{\sigma}}(\boldsymbol{u})=(\boldsymbol{\sigma}(\boldsymbol{u})$. $\boldsymbol{n})\left.\right|_{\Gamma_{\sigma}}$ and $R_{\Gamma_{D}}(\Phi)=\left.(\boldsymbol{\nabla} \Phi \cdot \boldsymbol{n})\right|_{\Gamma_{D}}$. We further introduce the sesquilinear forms

$$
\begin{aligned}
a(\boldsymbol{v}, \boldsymbol{w}) & :=\int_{\Omega} c_{i j k l} \varepsilon_{k l}(\boldsymbol{v}) \varepsilon_{i j}(\overline{\boldsymbol{w}}) d \boldsymbol{x} \\
b(\varphi, \boldsymbol{v}) & :=\int_{\Omega} e_{k i j} \frac{\partial \varphi}{\partial x_{k}} \varepsilon_{i j}(\overline{\boldsymbol{v}}) d \boldsymbol{x}, \\
c(\varphi, \psi) & :=\int_{\Omega} \epsilon_{i j} \frac{\partial \varphi}{\partial x_{i}} \frac{\partial \bar{\psi}}{\partial x_{j}} d \boldsymbol{x},
\end{aligned}
$$

where $\boldsymbol{v}, \boldsymbol{w} \in \boldsymbol{H}^{1}(\Omega)$ and $\varphi, \psi \in H^{1}(\Omega)$ with $\overline{\boldsymbol{w}}$ and $\bar{\psi}$ denoting the complex conjugation.

The variational formulation of the problem of piezoelectrically driven surface acoustic waves then reads:

Find $\boldsymbol{u} \in \boldsymbol{V}_{\Gamma_{u}}$ and $\Phi \in W_{\Gamma_{\Phi}}$ such that for all $\boldsymbol{v} \in \boldsymbol{V}$ and $\psi \in W$

$$
\begin{aligned}
a(\boldsymbol{u}, \boldsymbol{v})+b(\Phi, \boldsymbol{v})-\omega^{2}(\boldsymbol{u}, \boldsymbol{v})_{0, \Omega} & =\left\langle\boldsymbol{\sigma}_{n}, \boldsymbol{v}\right\rangle \\
b(\psi, \boldsymbol{u})-c(\Phi, \psi) & =\left\langle D_{n}, \psi\right\rangle
\end{aligned}
$$


Hereafter, $\langle\cdot, \cdot\rangle$ stands both for the dual pairing between $\boldsymbol{H}^{-\frac{1}{2}}\left(\Gamma_{\sigma}\right)$ and $\boldsymbol{H}_{00}^{\frac{1}{2}}\left(\Gamma_{\sigma}\right)$ and for the dual pairing between $H^{-\frac{1}{2}}\left(\Gamma_{D}\right)$ and $H_{00}^{\frac{1}{2}}\left(\Gamma_{D}\right)$.

The above sesquilinear forms define linear operators $\boldsymbol{A}: \boldsymbol{H}^{1}(\Omega) \rightarrow \boldsymbol{V}^{*}, \boldsymbol{B}: H^{1}(\Omega) \rightarrow \boldsymbol{V}^{*}$ and $\boldsymbol{C}: H^{1}(\Omega) \rightarrow$ $W^{*}$ so that $(18 \mathrm{a}),(18 \mathrm{~b})$ can be written in operator form as:

Find $\boldsymbol{u} \in \boldsymbol{V}$ and $\Phi \in W$ such that

$$
\begin{aligned}
\left(\boldsymbol{A}-\omega^{2} \boldsymbol{I}\right) \boldsymbol{u}+\boldsymbol{B} \Phi & =\mathbf{f}, \\
\boldsymbol{B}^{*} \boldsymbol{u}-\boldsymbol{C} \Phi & =g,
\end{aligned}
$$

where $\boldsymbol{I}$ stands for the injection $\boldsymbol{I}: \boldsymbol{V} \rightarrow \boldsymbol{V}^{*}$ and the right-hand sides $\mathbf{f} \in \boldsymbol{V}^{*}, g \in W^{*}$ are given by

$$
\begin{aligned}
\mathbf{f} & :=\boldsymbol{R}_{\Gamma_{\sigma}}^{*}\left(\boldsymbol{\sigma}_{n}\right)-\left(\boldsymbol{A}-\omega^{2} \boldsymbol{I}\right) \boldsymbol{E}_{\Gamma_{u}}\left(\boldsymbol{u}_{\Gamma}\right)-\boldsymbol{B} E_{\Gamma_{\Phi}}\left(\Phi_{\Gamma}\right), \\
g & :=R_{\Gamma_{D}}^{*}\left(D_{n}\right)-\boldsymbol{B}^{*} \boldsymbol{E}_{\Gamma_{u}}\left(\boldsymbol{u}_{\Gamma}\right)+\boldsymbol{C} E_{\Gamma_{\Phi}}\left(\Phi_{\Gamma}\right) .
\end{aligned}
$$

Lemma 4.1. The operators $\boldsymbol{A}, \boldsymbol{B}$ and $\boldsymbol{C}$ are bounded linear operators. Moreover, the operator $\boldsymbol{A}$ is symmetric and $\boldsymbol{V}$-elliptic, and the operator $\boldsymbol{C}$ is symmetric and $W$ elliptic.

Proof. The continuity of $\boldsymbol{A}, \boldsymbol{B}$ and $\boldsymbol{C}$ follows readily by applying the Cauchy Schwarz inequality. The symmetry of $\boldsymbol{A}$ results from the symmetry of the elasticity tensor $\boldsymbol{c}$, whereas the $\boldsymbol{V}$-ellipticity of $\boldsymbol{A}$ is a direct consequence of the positive definiteness of $\boldsymbol{c}$ and Korn's inequality. Likewise, the symmetry of $\boldsymbol{C}$ follows from the symmetry of the piezoelectric tensor $\boldsymbol{\epsilon}$ and the $W$-ellipticity can be deduced from the positive definiteness of $\epsilon$.

The invertibility of $C$ allows us to eliminate $\Phi$ from (19a),(19b) which results in the Schur complement system

$$
\boldsymbol{S u}-\omega^{2} \boldsymbol{I} \boldsymbol{u}=\boldsymbol{F} .
$$

Here, the Schur complement operator $\boldsymbol{S}: \boldsymbol{V} \rightarrow \boldsymbol{V}^{*}$ and the right-hand side $\boldsymbol{F}$ are given by

$$
\begin{aligned}
\boldsymbol{S} & :=\boldsymbol{A}+\boldsymbol{B} \boldsymbol{C}^{-1} \boldsymbol{B}^{*}, \\
\boldsymbol{F} & :=\mathbf{f}+\boldsymbol{B} \boldsymbol{C}^{-1} g .
\end{aligned}
$$

Lemma 4.2. The derived Schur complement operator $\boldsymbol{S}$ is a bounded, symmetric and $\boldsymbol{V}$-elliptic linear operator. Denoting by $\gamma_{A}$ and $\gamma_{C}$ the ellipticity constants of $\boldsymbol{A}$ and $\boldsymbol{C}$, respectively, for the ellipticity constant $\gamma_{S}$ of $\boldsymbol{S}$ and the norm $\|\boldsymbol{S}\|$ we have the estimates

$$
\|\boldsymbol{S}\| \leq\|\boldsymbol{A}\|+\frac{\|\boldsymbol{B}\|^{2}}{\gamma_{C}}, \quad \gamma_{S} \geq \gamma_{A} .
$$

Proof. The symmetry of $\boldsymbol{S}$ follows immediately from the symmetry of $\boldsymbol{A}$ and $\boldsymbol{C}$. Moreover, for $\boldsymbol{v}, \boldsymbol{w} \in \boldsymbol{V}$ we have

$$
\begin{aligned}
\langle\boldsymbol{S} \boldsymbol{v}, \boldsymbol{w}\rangle & =\langle\boldsymbol{A} \boldsymbol{v}, \boldsymbol{w}\rangle+\left\langle\boldsymbol{C}^{-1} \boldsymbol{B}^{*} \boldsymbol{v}, \boldsymbol{B}^{*} \boldsymbol{w}\right\rangle \\
& \leq\left(\|\boldsymbol{A}\|+\left\|\boldsymbol{C}^{-1}\right\|\|\boldsymbol{B}\|^{2}\right)\|\boldsymbol{v}\|_{1, \Omega}\|\boldsymbol{w}\|_{1, \Omega} .
\end{aligned}
$$

Hence, taking $\left\|\boldsymbol{C}^{-1}\right\| \leq \gamma_{C}^{-1}$ into account, this gives the upper bound for $\|\boldsymbol{S}\|$ in (24). The lower bound for $\gamma_{S}$ can be readily deduced from

$$
\langle\boldsymbol{S} \boldsymbol{v}, \boldsymbol{v}\rangle=\langle\boldsymbol{A} \boldsymbol{v}, \boldsymbol{v}\rangle+\left\langle\boldsymbol{C}^{-1} \boldsymbol{B}^{*} \boldsymbol{v}, \boldsymbol{B}^{*} \boldsymbol{v}\right\rangle \geq \gamma_{A}\|\boldsymbol{v}\|_{1, \Omega}^{2} .
$$

The Riesz Schauder theory of compact, self-adjoint linear operators is not directly applicable to (21), since the Schur complement operator $\boldsymbol{S}$ is not a self-adjoint endomorphism. Nevertheless, the Fredholm alternative holds true for (21), as can be seen by introducing the operator $\boldsymbol{S}_{R}^{-1}: \boldsymbol{L}^{2}(\Omega) \rightarrow \boldsymbol{V} \subset \boldsymbol{L}^{2}(\Omega)$ according to

$$
\boldsymbol{S}_{R}^{-1} \boldsymbol{v}:=\boldsymbol{S}^{-1} \boldsymbol{v}, \quad \boldsymbol{v} \in \boldsymbol{L}^{2}(\Omega) .
$$

Then, (21) can be rewritten as

$$
\boldsymbol{S u}-\omega^{2} \boldsymbol{u}=-\omega^{2} \boldsymbol{S}\left(\boldsymbol{S}_{R}^{-1}-\omega^{-2}\right) \boldsymbol{u}=\boldsymbol{F},
$$

and we obtain the following result:

\section{Theorem 4.1. (Fredholm Alternative)}

a) For $\omega^{2} \in \mathbb{R}$, exactly one of the following alternatives holds true:

(1) $\boldsymbol{u}=\mathbf{0}$ is the only solution of the eigenvalue problem $\boldsymbol{S} \boldsymbol{u}=\omega^{2} \boldsymbol{I} \boldsymbol{u}$. In this case, for every $\boldsymbol{F} \in \boldsymbol{V}^{*}$ the equation $\left(\boldsymbol{S}-\omega^{2} \boldsymbol{I}\right) \boldsymbol{u}=\boldsymbol{F}$ admits a unique solution $\boldsymbol{u} \in \boldsymbol{V}$ depending continuously on $\boldsymbol{F}$.

(2) There is a finite number $M$ of linear independent eigenfunctions $\boldsymbol{u}_{1}, \ldots \boldsymbol{u}_{M}$ satisfying $\boldsymbol{S} \boldsymbol{u}_{m}=$ $\omega^{2} \boldsymbol{I} \boldsymbol{u}_{m}$. In this case, if $\overline{\boldsymbol{u}}$ solves $\left(\boldsymbol{S}-\omega^{2} \boldsymbol{I}\right) \boldsymbol{u}=\boldsymbol{F}$ (i.e., if the equation is solvable), the general solution can be obtained with arbitrary $\alpha_{m} \in \mathbb{R}$ by

$$
\boldsymbol{u}=\overline{\boldsymbol{u}}+\sum_{m=1}^{M} \alpha_{m} \boldsymbol{u}_{m}
$$

b) The spectrum of $\boldsymbol{S}$ consists of a sequence of countably many real eigenvalues $0<\omega_{1}^{2}<\omega_{2}^{2}<\ldots$ tending to infinity, i.e., $\lim _{j \rightarrow \infty} \omega_{j}^{2}=\infty$.

c) If $\omega^{2} \in \mathbb{R}$ is an eigenvalue of $\boldsymbol{S}$, the equation $(\boldsymbol{S}-$ $\left.\omega^{2} \boldsymbol{I}\right) \boldsymbol{u}=\boldsymbol{F}$ is solvable if and only if $\boldsymbol{F} \in(\boldsymbol{S}-$ $\left.\omega^{2} \boldsymbol{I}\right)(\boldsymbol{V})$, i.e., iff $\boldsymbol{F} \in \operatorname{Ker}\left(\boldsymbol{S}-\omega^{2} \boldsymbol{I}\right)^{0}$ where

$$
\begin{aligned}
& \operatorname{Ker}\left(\boldsymbol{S}-\omega^{2} \boldsymbol{I}\right)^{0}:= \\
& \left\{\boldsymbol{v}^{*} \in \boldsymbol{V}^{*} \mid\left\langle\boldsymbol{v}^{*}, \boldsymbol{v}\right\rangle=0, \boldsymbol{v} \in \operatorname{Ker}\left(\boldsymbol{S}-\omega^{2} \boldsymbol{I}\right)\right\} .
\end{aligned}
$$

Proof. The operator $\boldsymbol{S}_{R}^{-1}$ as given by (25) is symmetric in $\boldsymbol{L}^{2}(\Omega)$. Indeed, using the symmetry of $\boldsymbol{S}$, for $\boldsymbol{v}, \boldsymbol{w} \in$ $\boldsymbol{L}^{2}(\Omega)$ we obtain

$$
\begin{aligned}
\left(\boldsymbol{S}_{R}^{-1} \boldsymbol{v}, \boldsymbol{w}\right)_{0, \Omega} & =\left(\boldsymbol{S}_{R}^{-1} \boldsymbol{v}, \boldsymbol{S} \boldsymbol{S}_{R}^{-1} \boldsymbol{w}\right)_{0, \Omega} \\
& =\left(\boldsymbol{S} \boldsymbol{S}_{R}^{-1} \boldsymbol{v}, \boldsymbol{S}_{R}^{-1} \boldsymbol{w}\right)_{0, \Omega}=\left(\boldsymbol{v}, \boldsymbol{S}_{R}^{-1} \boldsymbol{w}\right)_{0, \Omega} .
\end{aligned}
$$

It is bounded, since for $\boldsymbol{v} \in \boldsymbol{L}^{2}(\Omega)$ :

$$
\begin{aligned}
\gamma_{S}\left\|\boldsymbol{S}_{R}^{-1} \boldsymbol{v}\right\|_{0, \Omega}^{2} & \leq \gamma_{S}\left\|\boldsymbol{S}_{R}^{-1} \boldsymbol{v}\right\|_{1 ; \Omega}^{2} \leq\left\langle\boldsymbol{S} \boldsymbol{S}_{R}^{-1} \boldsymbol{v}, \boldsymbol{S}_{R}^{-1} \boldsymbol{v}\right\rangle \\
& =\left(\boldsymbol{v}, \boldsymbol{S}_{R}^{-1} \boldsymbol{v}\right)_{0, \Omega} \leq\|\boldsymbol{v}\|_{0, \Omega}\left\|\boldsymbol{S}_{R}^{-1} \boldsymbol{v}\right\|_{0, \Omega}
\end{aligned}
$$

Moreover, for a generalized eigenvalue $\omega^{2} \neq 0$ and a corresponding eigenfunction $\boldsymbol{u} \in \boldsymbol{V}$ of $\boldsymbol{S}$, the operator $S_{R}^{-1}$ satisfies the inverse eigenvalue problem

$$
\boldsymbol{S}_{R}^{-1} \boldsymbol{u}=\frac{1}{\omega^{2}} \boldsymbol{u}
$$


On the other hand, if $\boldsymbol{u} \in \boldsymbol{L}^{2}(\Omega)$ satisfies (27), then $\boldsymbol{u} \in$ $\boldsymbol{V}$ (since $\boldsymbol{S}_{R}^{-1}\left(\boldsymbol{L}^{2}(\Omega)\right) \subset \boldsymbol{V}$ ) and $\boldsymbol{u}$ is an eigenfunction of $\boldsymbol{S}$. The operator $\boldsymbol{S}_{R}^{-1}$ is compact, since the embedding $\boldsymbol{V} \subset \boldsymbol{L}^{2}(\Omega)$ is compact. Consequently, $\boldsymbol{S}_{R}^{-1}$ is a compact self-adjoint endomorphism on $\boldsymbol{L}^{2}(\Omega)$ and hence, in view of (26), the assertions can be deduced from the HilbertSchmidt theory and the Fredholm alternative (cf., e.g., [60]).

Theorem 4.1 tells us that the solvability of the Schur complement system (21) is guaranteed for almost all $\omega^{2} \in \mathbb{R}$. If $\omega^{2}$ is a generalized eigenvalue of $\boldsymbol{S}$, the solvability condition c) is in fact a condition on the boundary data, since $\boldsymbol{F}=\mathbf{f}+\boldsymbol{B} \boldsymbol{C}^{-1} g$ and by (20),

$$
\boldsymbol{F}=\boldsymbol{R}_{\Gamma_{\sigma}}^{*}\left(\boldsymbol{\sigma}_{n}\right)+\boldsymbol{B} \boldsymbol{C}^{-1} R_{\Gamma_{D}}^{*} D_{n}-\left(\boldsymbol{S}-\omega^{2} \boldsymbol{I}\right) \boldsymbol{E}_{\Gamma_{u}}\left(\boldsymbol{u}_{\Gamma}\right) .
$$

Remark 4.1 (A 2.5 D Model). SAWs propagate along the surface of a substrate. To be more precise, the amplitude of the displacement $\boldsymbol{u}$ decays exponentially with the depth of the penetration into the substrate [35]. In true Rayleigh waves, most of the energy (90\%) is concentrated within one wavelength from the surface. If $h$ is the height of the substrate with the surface located at $x_{3}=0$, this leads to the definition

$$
H\left(x_{3}\right):=\frac{1-e^{\frac{\alpha\left(x_{3}+h\right)}{h}}}{1-e^{\alpha}} .
$$

We reduce the dependency on $x_{3}$ to exponential decay:

$$
\begin{aligned}
& \boldsymbol{u}(\boldsymbol{x})=H\left(x_{3}\right) \boldsymbol{u}\left(x_{1}, x_{2}\right), \\
& \Phi(\boldsymbol{x})=H\left(x_{3}\right) \Phi\left(x_{1}, x_{2}\right) .
\end{aligned}
$$

The factor $\alpha$ measures the rapidity of the exponential decay. A good guess for this parameter can be obtained from $2 \mathrm{D}$ calculations.

\section{Finite element approximation of the surface acoustic wave equations}

We now restrict ourselves to the case where the computational domain $\Omega \subset \mathbb{R}^{d}$ is a polygonal resp. polyhedral domain. We provide a simplicial triangulation $\mathcal{T}_{h}(\Omega)$ of $\Omega$ that aligns with $\Gamma_{u}$ and $\Gamma_{\phi}$ and denote by $S_{0, \Gamma^{\prime}}^{(k)}\left(\Omega ; \mathcal{T}_{h}(\Omega)\right), k \in \mathbb{N}, \Gamma^{\prime}=\Gamma_{u}$ or $\Gamma^{\prime}=\Gamma_{\Phi}$ the finite element space of continuous functions $v_{h}: \Omega \rightarrow \mathbb{C}$ vanishing on $\Gamma^{\prime}$ and satisfying $\left.v_{h}\right|_{T} \in P_{k}(T), T \in \mathcal{T}_{h}(\Omega)$, where $P_{k}(T)$ stands for the linear space of complex valued polynomials of degree $k$ on $T \in \mathcal{T}_{h}(\Omega)$. We approximate the space $\boldsymbol{V}$ of displacements and the space $W$ of electric potentials by

$$
\begin{aligned}
\boldsymbol{V}_{h} & :=S_{0, \Gamma_{u}}^{(k)}\left(\Omega ; \mathcal{T}_{h}(\Omega)\right)^{d} \\
W_{h} & :=S_{0, \Gamma_{\Phi}}^{(k)}\left(\Omega ; \mathcal{T}_{h}(\Omega)\right) .
\end{aligned}
$$

We refer to $\boldsymbol{A}_{h}: \boldsymbol{V}_{h} \rightarrow \boldsymbol{V}_{h}^{*}, \boldsymbol{B}_{h}: W_{h} \rightarrow \boldsymbol{V}_{h}^{*}$ and $\boldsymbol{C}_{h}:$ $W_{h} \rightarrow W_{h}^{*}$ as the operators associated with the sesquilinear forms $a(\cdot, \cdot), b(\cdot, \cdot)$ and $c(\cdot, \cdot)$ when restricted to the respective finite dimensional subspaces, i.e., $\left.a\right|_{\boldsymbol{V}_{h} \times \boldsymbol{V}_{h}}$, $\left.b\right|_{W_{h} \times \boldsymbol{V}_{h}}$, and $\left.c\right|_{W_{h} \times W_{h}}$. We note that these operators inherit their properties from its continuous counterparts. In particular, $\boldsymbol{A}_{h}, \boldsymbol{B}_{h}$ and $\boldsymbol{C}_{h}$ are bounded linear operators. Moreover, the operator $\boldsymbol{A}_{h}$ is symmetric and $\boldsymbol{V}_{h^{-}}$ elliptic, whereas $\boldsymbol{C}_{h}$ is symmetric and $W_{h}$-elliptic having the same ellipticity constants $\gamma_{A}$ and $\gamma_{C}$. We further define $\mathbf{f}_{h} \in \boldsymbol{V}_{h}^{*}$ and $g_{h} \in W_{h}^{*}$ by $\left\langle\mathbf{f}_{h}, \boldsymbol{u}_{h}\right\rangle:=\left\langle\mathbf{f}, \boldsymbol{u}_{h}\right\rangle, \boldsymbol{u}_{h} \in$ $\boldsymbol{V}_{h}$, and $\left\langle g_{h}, \Phi_{h}\right\rangle:=\left\langle g, \Phi_{h}\right\rangle, \Phi_{h} \in W_{h}$.

The finite element approximation of (19) amounts now to the computation of $\boldsymbol{u}_{h} \in \boldsymbol{V}_{h}$ and $\Phi_{h} \in W_{h}$ such that

$$
\begin{aligned}
\left(\boldsymbol{A}_{h}-\omega^{2} \boldsymbol{I}_{h}\right) \boldsymbol{u}_{h}+\boldsymbol{B}_{h} \Phi_{h} & =\mathbf{f}_{h}, \\
\boldsymbol{B}_{h}^{*} \boldsymbol{u}_{h}-\boldsymbol{C}_{h} \Phi_{h} & =g_{h},
\end{aligned}
$$

where $\boldsymbol{I}_{h}$ is the injection $\boldsymbol{I}_{h}: \boldsymbol{V}_{h} \rightarrow \boldsymbol{V}_{h}^{*}$.

Again, static condensation of $\Phi_{h}$ yields the discrete Schur complement system

$$
\left(\boldsymbol{S}_{h}-\omega^{2} \boldsymbol{I}_{h}\right) \boldsymbol{u}_{h}=\boldsymbol{F}_{h}
$$

with $\boldsymbol{F}_{h}:=\mathbf{f}_{h}+\boldsymbol{B}_{h} \boldsymbol{C}_{h}^{-1} g_{h}$ and the discrete Schur complement operator $\boldsymbol{S}_{h}$ given by

$$
\boldsymbol{S}_{h}:=\boldsymbol{A}_{h}+\boldsymbol{B}_{h} \boldsymbol{C}_{h}^{-1} \boldsymbol{B}_{h}^{*} .
$$

It is an easy exercise to show that $\boldsymbol{S}_{h}$ as given by (34) is indeed the Galerkin approximation of $\boldsymbol{S}$, i.e.,

$$
\left\langle\boldsymbol{S}_{h} \boldsymbol{v}_{h}, \boldsymbol{w}_{h}\right\rangle=\left\langle\boldsymbol{S} \boldsymbol{v}_{h}, \boldsymbol{w}_{h}\right\rangle, \boldsymbol{v}_{h}, \boldsymbol{w}_{h} \in \boldsymbol{V}_{h} .
$$

If $\omega \in \mathbb{R}$ is such that (21) is solvable, then it is wellknown that the operator $\boldsymbol{S}_{\omega}:=\boldsymbol{S}-\omega^{2} \boldsymbol{I}$ satisfies the inf-sup condition (cf., e.g., [12])

$$
\inf _{\mathbf{0} \neq \boldsymbol{v} \in \boldsymbol{V}} \sup _{\mathbf{0} \neq \boldsymbol{w} \in \boldsymbol{V}} \frac{\left|\left\langle\boldsymbol{S}_{\omega} \boldsymbol{v}, \boldsymbol{w}\right\rangle\right|}{\|\boldsymbol{v}\|_{1, \Omega}\|\boldsymbol{w}\|_{1, \Omega}} \geq \beta>0 .
$$

As has been shown in [31], for sufficiently small $h$ a discrete inf-sup condition holds true as well:

Theorem 5.1. Let $\boldsymbol{S}_{h}$ be given by (34) and assume that for some $\omega \in \mathbb{R}$ the operator $\boldsymbol{S}_{\omega}$ satisfies the inf-sup condition (35). Then, there exist $h_{0}>0$ and $\beta_{\text {min }}>0$ such that for all $h \leq h_{0}$ the operator $\boldsymbol{S}_{h, \omega}:=\boldsymbol{S}_{h}-\omega^{2} \boldsymbol{I}_{h}$ satisfies the discrete inf-sup condition

$$
\inf _{\mathbf{0} \neq \boldsymbol{v}_{h} \in \boldsymbol{V}_{h}} \sup _{\mathbf{0} \neq \boldsymbol{w}_{h} \in \boldsymbol{V}_{h}} \frac{\left|\left\langle\boldsymbol{S}_{h, \omega} \boldsymbol{v}_{h}, \boldsymbol{w}_{h}\right\rangle\right|}{\left\|\boldsymbol{v}_{h}\right\|_{1, \Omega}\left\|\boldsymbol{w}_{h}\right\|_{1, \Omega}} \geq \beta_{h} \geq \beta_{\text {min }} .
$$

Proof. We give the proof for completeness and assume without restriction of generality that $\omega \neq 0$. We introduce bounded linear operators $\boldsymbol{S}^{-1}: \boldsymbol{V} \rightarrow \boldsymbol{V}$ and $\boldsymbol{S}_{h}^{-1}$ : $\boldsymbol{V}_{h} \rightarrow \boldsymbol{V}_{h}$ by $\boldsymbol{S}\left(\boldsymbol{S}^{-1} \boldsymbol{v}\right)=\boldsymbol{I} \boldsymbol{v}, \boldsymbol{v} \in \boldsymbol{V}$, and $\boldsymbol{S}_{h}\left(\boldsymbol{S}_{h}^{-1} \boldsymbol{v}_{h}\right)=$ $\boldsymbol{I}_{h} \boldsymbol{v}_{h}, \boldsymbol{v}_{h} \in \boldsymbol{V}_{h}$. Taking (35) into account, for $\boldsymbol{v} \in \boldsymbol{V}$ we then get

$$
\begin{aligned}
\beta\|\boldsymbol{v}\|_{1 ; \Omega} & \leq \sup _{\mathbf{0} \neq \boldsymbol{w} \in \boldsymbol{V}} \frac{\left|\left\langle\boldsymbol{S}_{\omega} \boldsymbol{v}, \boldsymbol{w}\right\rangle\right|}{\|\boldsymbol{w}\|_{1 ; \Omega}}= \\
& =\sup _{\mathbf{0} \neq \boldsymbol{w} \in \boldsymbol{V}} \frac{\mid\left\langle\boldsymbol{S}\left(\boldsymbol{v}-\omega^{2} \boldsymbol{S}^{-1} \boldsymbol{v}, \boldsymbol{w}\right\rangle\right|}{\|\boldsymbol{w}\|_{1 ; \Omega}} \\
& \leq\|\boldsymbol{S}\|\left\|\boldsymbol{v}-\omega^{2} \boldsymbol{S}^{-1} \boldsymbol{v}\right\|_{1 ; \Omega} .
\end{aligned}
$$


Hence, in view of the $\boldsymbol{V}_{h}$-ellipticity of $\boldsymbol{S}_{h}$ for $\boldsymbol{v}_{h} \in \boldsymbol{V}_{h}$ we obtain

$$
\begin{aligned}
& \sup _{\mathbf{0} \neq \boldsymbol{w}_{h} \in \boldsymbol{V}_{h}} \frac{\left|\left\langle\boldsymbol{S}_{h, \omega} \boldsymbol{v}_{h}, \boldsymbol{w}_{h}\right\rangle\right|}{\left\|\boldsymbol{w}_{h}\right\|_{1 ; \Omega}}= \\
= & \sup _{\mathbf{0} \neq \boldsymbol{w}_{h} \in \boldsymbol{V}_{h}} \frac{\left|\left\langle\boldsymbol{S}_{h}\left(\boldsymbol{v}_{h}-\omega^{2} \boldsymbol{S}_{h}^{-1} \boldsymbol{v}_{h}\right), \boldsymbol{w}_{h}\right\rangle\right|}{\left\|\boldsymbol{w}_{h}\right\|_{1 ; \Omega}} \\
\geq & \frac{\left|\left\langle\boldsymbol{S}_{h}\left(\boldsymbol{v}_{h}-\omega^{2} \boldsymbol{S}_{h}^{-1} \boldsymbol{v}_{h}\right), \boldsymbol{v}_{h}-\omega^{2} \boldsymbol{S}_{h}^{-1} \boldsymbol{v}_{h}\right\rangle\right|}{\left\|\boldsymbol{v}_{h}-\omega^{2} \boldsymbol{S}_{h}^{-1} \boldsymbol{v}_{h}\right\|_{1 ; \Omega}} \\
\geq & \gamma_{S}\left\|\boldsymbol{v}_{h}-\omega^{2} \boldsymbol{S}_{h}^{-1} \boldsymbol{v}_{h}\right\|_{1 ; \Omega} \\
\geq & \gamma_{S}\left\|\boldsymbol{v}_{h}-\omega^{2} \boldsymbol{S}^{-1} \boldsymbol{v}_{h}\right\|_{1 ; \Omega}-\omega^{2} \gamma_{S}\left\|\left(\boldsymbol{S}_{h}^{-1}-\boldsymbol{S}^{-1}\right) \boldsymbol{v}_{h}\right\|_{1 ; \Omega} \\
\geq & \left(\frac{\beta \gamma_{S}}{\|\boldsymbol{S}\|}-\omega^{2} \gamma_{S}\left\|\boldsymbol{S}_{h}^{-1}-\boldsymbol{S}^{-1}\right\|\right)\left\|\boldsymbol{v}_{h}\right\|_{1 ; \Omega}
\end{aligned}
$$

whence

$$
\beta_{h} \geq \frac{\beta \gamma_{S}}{\|\boldsymbol{S}\|}-\omega^{2} \gamma_{S}\left\|\boldsymbol{S}_{h}^{-1}-\boldsymbol{S}^{-1}\right\|
$$

Since $\left\|\boldsymbol{S}_{h}^{-1}-\boldsymbol{S}^{-1}\right\| \rightarrow 0$ as $h \rightarrow 0$, we conclude.

\section{Multilevel preconditioned iterative solution of the saddle point system}

The discrete system (32) represents an algebraic saddle point problem of the form

$$
\left(\begin{array}{cc}
\boldsymbol{A} & \boldsymbol{B} \\
\boldsymbol{B}^{T} & -\boldsymbol{C}
\end{array}\right)\left(\begin{array}{l}
\boldsymbol{u} \\
\boldsymbol{\Phi}
\end{array}\right)=\left(\begin{array}{l}
\boldsymbol{f} \\
\boldsymbol{g}
\end{array}\right) \quad \text { or } \quad \boldsymbol{Z} \boldsymbol{U}=\boldsymbol{\ell}
$$

Here, $\boldsymbol{A} \in \mathbb{R}^{n \times n}$ and $\boldsymbol{C} \in \mathbb{R}^{m \times m}$ are symmetric, positive definite matrices satisfying

$$
\begin{aligned}
\gamma_{1} \boldsymbol{v}^{T} \boldsymbol{v} \leq \boldsymbol{v}^{T} \boldsymbol{A} \boldsymbol{v} \leq \Gamma_{1} \boldsymbol{v}^{T} \boldsymbol{v}, \quad \boldsymbol{v} \in \mathbb{R}^{n} \\
\gamma_{2} \boldsymbol{\Phi}^{T} \boldsymbol{\Phi} \leq \boldsymbol{\Phi}^{T} \boldsymbol{C} \boldsymbol{\Phi} \leq \Gamma_{2} \boldsymbol{\Phi}^{T} \boldsymbol{\Phi}, \quad \boldsymbol{\Phi} \in \mathbb{R}^{m}
\end{aligned}
$$

with constants $0<\gamma_{i} \leq \Gamma_{i}, 1 \leq i \leq 2$. Moreover, $\boldsymbol{B} \in$ $\mathbb{R}^{n \times m}$ and $\boldsymbol{f} \in \mathbb{R}^{n}, \boldsymbol{g} \in \mathbb{R}^{m}$ whence $\boldsymbol{Z} \in \mathbb{R}^{N \times N}, \boldsymbol{\ell} \in \mathbb{R}^{N}$ where $N:=n+m$. We further assume that $\boldsymbol{Z}$ satisfies

$$
\inf _{\boldsymbol{U} \neq \mathbf{0}} \sup _{\boldsymbol{V} \neq \mathbf{0}} \frac{\left|\boldsymbol{V}^{T} \boldsymbol{Z} \boldsymbol{U}\right|}{\|\boldsymbol{U}\|\|\boldsymbol{V}\|} \geq \gamma_{Z}>0
$$

where $\|\cdot\|$ stands for the Euclidean norm in $\mathbb{R}^{N}$.

Generalized saddle point problems such as (36) arise in many applications as, for instance, in the framework of stabilized Stokes systems $[49,50]$ or in mixed finite element approximations of boundary value problems for elliptic equations and systems [12]. We refer to $[7,15$, $38]$ and to the references therein for basic results and to $[5,10,14,17,25,26,31,32]$ for efficient iterative solution techniques including multilevel preconditioning.

In the sequel, we closely follow $[32,50]$ and consider block-diagonal preconditioners of the form

$$
P^{-1}:=\left(\begin{array}{cc}
\tilde{A} & \mathbf{0} \\
\mathbf{0} & \tilde{C}
\end{array}\right)
$$

where we assume that $\tilde{\boldsymbol{A}} \in \mathbb{R}^{n \times n}$ and $\tilde{\boldsymbol{C}} \in \mathbb{R}^{m \times m}$ are symmetric, positive definite matrices satisfying

$$
\begin{gathered}
\tilde{\gamma}_{1} \boldsymbol{v}^{T} \boldsymbol{v} \leq \boldsymbol{v}^{T} \tilde{\boldsymbol{A}} \boldsymbol{v} \leq \tilde{\Gamma}_{1} \boldsymbol{v}^{T} \boldsymbol{v}, \quad \boldsymbol{v} \in \mathbb{R}^{n}, \\
\tilde{\gamma}_{2} \boldsymbol{\Phi}^{T} \boldsymbol{\Phi} \leq \boldsymbol{\Phi}^{T} \tilde{\boldsymbol{C}} \boldsymbol{\Phi} \leq \tilde{\Gamma}_{2} \boldsymbol{\Phi}^{T} \boldsymbol{\Phi}, \quad \boldsymbol{\Phi} \in \mathbb{R}^{m}
\end{gathered}
$$

with constants $0<\tilde{\gamma}_{i} \leq \tilde{\Gamma}_{i}, 1 \leq i \leq 2$.

As an easy consequence from (40) we deduce that $\boldsymbol{P}^{-1}$ is positive definite with

$$
\Gamma_{P}^{-1} \boldsymbol{z}^{T} \boldsymbol{z} \leq \boldsymbol{z}^{T} \boldsymbol{P}^{-1} \boldsymbol{z} \leq \gamma_{P}^{-1} \boldsymbol{z}^{T} \boldsymbol{z}, \quad \boldsymbol{z} \in \mathbb{R}^{N},
$$

where $\gamma_{P}^{-1}:=\max \left(\tilde{\Gamma}_{1}, \tilde{\Gamma}_{2}\right)$ and $\Gamma_{P}^{-1}:=1 /\left(\min \left(\tilde{\gamma}_{1}, \tilde{\gamma}_{2}\right)\right.$.

Using (38) and (41), we can readily derive lower and upper bounds for the spectrum of the preconditioned matrix $\boldsymbol{P}^{\frac{1}{2}} \boldsymbol{Z} \boldsymbol{P}^{\frac{1}{2}}$ :

Theorem 6.1. Suppose that (38) and (41) are satisfied for $\boldsymbol{Z}$ and $\boldsymbol{P}^{-1}$, respectively. Then, for $\boldsymbol{V} \in \mathbb{R}^{N}$ there holds

$$
\gamma_{P Z} \boldsymbol{V}^{T} \boldsymbol{V} \leq \boldsymbol{V}^{T} \boldsymbol{P}^{\frac{1}{2}} \boldsymbol{Z} \boldsymbol{P}^{\frac{1}{2}} \boldsymbol{V} \leq \Gamma_{P Z} \boldsymbol{V}^{T} \boldsymbol{V}
$$

where $\gamma_{P Z}:=\gamma_{P} \gamma_{Z}$ and $\Gamma_{P Z}:=\Gamma_{P}\|\boldsymbol{Z}\|$.

Proof. Straightforward computations yield

$$
\begin{aligned}
& \inf _{\boldsymbol{W} \neq \mathbf{0}} \sup _{\boldsymbol{V} \neq \mathbf{0}} \frac{\boldsymbol{V}^{T} \boldsymbol{P}^{\frac{1}{2}} \boldsymbol{Z} \boldsymbol{P}^{\frac{1}{2}} \boldsymbol{W}}{\|\boldsymbol{V}\|\|\boldsymbol{W}\|}= \\
& =\inf _{\tilde{\boldsymbol{W}} \neq \mathbf{0}} \sup _{\tilde{\boldsymbol{V}} \neq \mathbf{0}} \frac{\tilde{\boldsymbol{V}}^{T} \boldsymbol{Z} \tilde{\boldsymbol{W}}}{\left(\tilde{\boldsymbol{V}}^{T} \boldsymbol{P}^{-1} \tilde{\boldsymbol{V}}\right)^{\frac{1}{2}}\left(\tilde{\boldsymbol{W}}^{T} \boldsymbol{P}^{-1} \tilde{\boldsymbol{W}}\right)^{\frac{1}{2}}} \geq \\
& \geq \gamma_{P} \inf _{\tilde{\boldsymbol{W}} \neq \mathbf{0}} \sup _{\tilde{\boldsymbol{V}} \neq \mathbf{0}} \frac{\tilde{\boldsymbol{V}}^{T} \boldsymbol{Z} \tilde{\boldsymbol{W}}}{\|\tilde{\boldsymbol{V}}\|\|\tilde{\boldsymbol{W}}\|} \geq \gamma_{P} \gamma_{Z} .
\end{aligned}
$$

Similar arguments result in the upper bound in (42).

We allow the following inf-sup condition for the sesquilinear form $b(\cdot, \cdot)$ restricted to $\boldsymbol{V}_{h} \times W_{h}$ :

$$
\inf _{\boldsymbol{v}_{h} \in \boldsymbol{V}_{h}} \sup _{\varphi_{h} \in W_{h}} \frac{\left|b\left(\boldsymbol{v}_{h}, \varphi_{h}\right)\right|}{\left\|\boldsymbol{v}_{h}\right\| \boldsymbol{V}\left\|\varphi_{h}\right\|_{W}} \geq \beta_{h} \geq \beta_{\min } \geq 0
$$

i.e., $\beta_{\min }=0$ is admitted. In this case, the associated matrix $\boldsymbol{B}$ may have a non-trivial kernel, and we get:

Lemma 6.1. Under the assumptions (37b) and (43), for $\boldsymbol{v} \in \mathbb{R}^{n}, \boldsymbol{v} \neq \mathbf{0}$ there holds

$$
\frac{\beta_{\min }^{2}}{\|\boldsymbol{C}\|} \leq \frac{\boldsymbol{v}^{T} \boldsymbol{B} \boldsymbol{C}^{-1} \boldsymbol{B}^{T} \boldsymbol{v}}{\boldsymbol{v}^{T} \boldsymbol{v}} \leq \frac{\|\boldsymbol{B}\|^{2}}{\gamma_{2}} .
$$

Proof. For $\boldsymbol{v} \in \mathbb{R}^{n}$ we readily obtain

$$
\begin{aligned}
\boldsymbol{v}^{T} \boldsymbol{B} \boldsymbol{C}^{-1} \boldsymbol{B}^{T} \boldsymbol{v} & =\sup _{\boldsymbol{\Phi} \neq \mathbf{0}} \frac{\left(\boldsymbol{\Phi}^{T} \boldsymbol{C} \boldsymbol{C}^{-1} \boldsymbol{B}^{T} \boldsymbol{v}\right)^{2}}{\boldsymbol{\Phi}^{T} \boldsymbol{C} \boldsymbol{\Phi}} \\
& \geq \frac{1}{\|\boldsymbol{C}\|} \frac{\left(\boldsymbol{\Phi}^{T} \boldsymbol{B} \boldsymbol{v}\right)^{2}}{\boldsymbol{\Phi}^{T} \boldsymbol{\Phi}} \geq \frac{\beta_{\min }^{2}}{\|\boldsymbol{C}\|} \boldsymbol{v}^{T} \boldsymbol{v}
\end{aligned}
$$

and

$$
\boldsymbol{v}^{T} \boldsymbol{B} \boldsymbol{C}^{-1} \boldsymbol{B}^{T} \boldsymbol{v} \leq \frac{1}{\gamma_{2}}\left\|\boldsymbol{B}^{T} \boldsymbol{v}\right\|^{2} \leq \frac{\|\boldsymbol{B}\|^{2}}{\gamma_{2}} \boldsymbol{v}^{T} \boldsymbol{v}
$$


The preconditioned saddle point system is given by

$$
\left(\begin{array}{cc}
\tilde{\boldsymbol{A}}^{-1} \boldsymbol{A} & \tilde{\boldsymbol{A}}^{-1} \boldsymbol{B} \\
\tilde{\boldsymbol{C}}^{-1} \boldsymbol{B}^{T} & -\tilde{\boldsymbol{C}}^{-1} \boldsymbol{C}
\end{array}\right)\left(\begin{array}{c}
\boldsymbol{u} \\
\boldsymbol{\Phi}
\end{array}\right)=\left(\begin{array}{c}
\tilde{\boldsymbol{A}}^{-1} \boldsymbol{f} \\
\tilde{\boldsymbol{C}}^{-1} \boldsymbol{g}
\end{array}\right) .
$$

A simple computation shows that the Schur complement matrix of the preconditioned system is given by

$$
\tilde{\boldsymbol{S}}=\tilde{\boldsymbol{A}}^{-1} \boldsymbol{S},
$$

thus completely neglecting the dependence on the preconditioner part $\tilde{\boldsymbol{C}}^{-1}$. This is immediately clear, since this preconditioner only speeds up the inner iteration when solving systems with coefficient matrix $\boldsymbol{C}$. The spectrum of $\tilde{\boldsymbol{A}}^{-1} \boldsymbol{S}$ can be determined from the eigenvalues of $\tilde{\boldsymbol{A}}^{-\frac{1}{2}} \boldsymbol{S} \tilde{\boldsymbol{A}}^{-\frac{1}{2}}$.

Theorem 6.2. For the spectrum of $\tilde{\boldsymbol{S}}$ we have the following lower and upper bound

$$
\gamma_{P S} \leq \frac{\boldsymbol{v}^{T} \tilde{\boldsymbol{S}} \boldsymbol{v}}{\|\boldsymbol{v}\|^{2}} \leq \Gamma_{P S}
$$

where

$$
\begin{aligned}
\gamma_{P S} & :=\frac{1}{\|\tilde{\boldsymbol{A}}\|}\left(\gamma_{1}+\frac{\beta_{\min }^{2}}{\|\boldsymbol{C}\|}\right), \\
\Gamma_{P S} & :=\frac{1}{\tilde{\gamma}_{1}}\left(\|\boldsymbol{A}\|+\frac{\|\boldsymbol{B}\|^{2}}{\gamma_{2}}\right) .
\end{aligned}
$$

Proof. Setting $\boldsymbol{w}:=\tilde{\boldsymbol{A}}^{-\frac{1}{2}} \boldsymbol{v}$, we obtain

$$
\begin{aligned}
& \frac{\boldsymbol{v}^{T} \tilde{\boldsymbol{A}}^{-\frac{1}{2}} \boldsymbol{S} \tilde{\boldsymbol{A}}^{-\frac{1}{2}} \boldsymbol{v}}{\boldsymbol{v}^{T} \boldsymbol{v}}= \\
& =\frac{\boldsymbol{v}^{T} \tilde{\boldsymbol{A}}^{-\frac{1}{2}} \boldsymbol{A} \tilde{\boldsymbol{A}}^{-\frac{1}{2}} \boldsymbol{v}}{\boldsymbol{v}^{T} \boldsymbol{v}}+\frac{\boldsymbol{v}^{T} \tilde{\boldsymbol{A}}^{-\frac{1}{2}} \boldsymbol{B} \boldsymbol{C}^{-1} \boldsymbol{B}^{T} \tilde{\boldsymbol{A}}^{-\frac{1}{2}} \boldsymbol{v}}{\boldsymbol{v}^{T} \boldsymbol{v}} \\
& =\frac{\boldsymbol{w}^{T} \boldsymbol{A} \boldsymbol{w}}{\boldsymbol{w}^{T} \tilde{\boldsymbol{A}} \boldsymbol{w}}+\frac{\boldsymbol{w}^{T} \boldsymbol{B} \boldsymbol{C}^{-1} \boldsymbol{B}^{T} \boldsymbol{w}}{\boldsymbol{w}^{T} \tilde{\boldsymbol{A}} \boldsymbol{w}}
\end{aligned}
$$

The first term can be estimated by the ellipticity properties of $\boldsymbol{A}$ and $\tilde{\boldsymbol{A}}$,

$$
\frac{\gamma_{1}}{\|\tilde{\boldsymbol{A}}\|} \leq \frac{\boldsymbol{w}^{T} \boldsymbol{A} \boldsymbol{w}}{\boldsymbol{w}^{T} \tilde{\boldsymbol{A}} \boldsymbol{w}} \leq \frac{\|\boldsymbol{A}\|}{\tilde{\gamma}_{1}}
$$

By Lemma 6.1 and the ellipticity property of $\tilde{\boldsymbol{A}}$, for the second term it follows that

$$
\frac{\beta_{\min }^{2}}{\|\tilde{\boldsymbol{A}}\|\|\boldsymbol{C}\|} \leq \frac{\boldsymbol{w}^{T} \boldsymbol{B} \boldsymbol{C}^{-1} \boldsymbol{B}^{T} \boldsymbol{w}}{\boldsymbol{w}^{T} \tilde{\boldsymbol{A}} \boldsymbol{w}} \leq \frac{\|\boldsymbol{B}\|^{2}}{\tilde{\gamma}_{1} \gamma_{2}} .
$$

Combining both estimates, gives the assertion.

We are particularly interested in such preconditioners where the lower and upper bounds $\gamma_{P Z}$ and $\Gamma_{P Z}$ for the spectrum of the preconditioned saddle point matrix $\boldsymbol{P} \boldsymbol{Z}$ and the corresponding bounds $\gamma_{P S}$ and $\Gamma_{P S}$ for the spectrum of the preconditioned Schur complement $\tilde{\boldsymbol{A}}^{-1} \boldsymbol{S}$ as well as the bounds for the spectrum of $\tilde{\boldsymbol{C}}^{-1} \boldsymbol{C}$ are independent of the granularity $h$ of the triangulations. Such preconditioners are provided by multilevel preconditioners of BPX-type with respect to a nested hierarchy of simplicial triangulations of the computational domain $\Omega$ (cf., e.g., [11,41]).

\section{Numerical Results}

In this section, we present a documentation of numerical results both for a test problem in order to study the performance of the block-diagonally preconditioned iterative solver of the finite element discretized surface acoustic wave equations and for a realistic surface acoustic wave device as it is used on microfluidic biochips. The solver is implemented within the finite element toolbox ALBERTA $[47,48]$.

\subsection{Test Problem}

For the test problem, we assume that, in Voigt notation, the elasticity tensor $\boldsymbol{c}=\left(c_{I K}\right)$ and the piezoelectric tensor $\boldsymbol{e}=\left(e_{I K}\right)$ are given by

$$
\begin{gathered}
\boldsymbol{c}=\left(\begin{array}{rrrrrr}
20 & 5 & 7 & 1 & 0 & 0 \\
5 & 20 & 7 & -1 & 0 & 0 \\
7 & 7 & 25 & 0 & 0 & 0 \\
-1 & -1 & 0 & 5 & 0 & 0 \\
0 & 0 & 0 & 0 & 6 & 1 \\
0 & 0 & 0 & 0 & 1 & 7
\end{array}\right), \\
\boldsymbol{e}=\left(\begin{array}{rrrrrr}
-4 & 1 & 1 & 0 & 0 & 0 \\
0 & -4 & 1 & 0 & 0 & 0 \\
1 & 1 & 1 & 1 & 1 & 1
\end{array}\right)
\end{gathered}
$$

whereas the dielectric tensor $\boldsymbol{\epsilon}=\left(\epsilon_{i j}\right)$ is chosen according to

$$
\boldsymbol{\epsilon}=\left(\begin{array}{ccc}
10 & 0 & 0 \\
0 & 10 & 0 \\
0 & 0 & 5
\end{array}\right)
$$

We note that the material moduli resemble the properties of a typical piezoelectric material like quartz.

The right-hand sides and boundary data are chosen in such a way that

$$
\boldsymbol{u}(\boldsymbol{x})=\boldsymbol{x}^{T} \boldsymbol{x}(1,2,3)^{T} \quad, \quad \Phi(\boldsymbol{x})=\sum_{i=1}^{3} \sin \left(\pi x_{i}\right)
$$

solves the time-harmonic surface acoustic wave equations (11),(12) and (15) for angular frequencies $\omega$ ranging between $\omega=0$ and $\omega=25$.

We apply the iterative solvers GMRES and BICGSTAB with and without the block-diagonal preconditioners to the algebraic saddle point problems arising from finite element discretizations with respect to uniform hierarchies of simplicial triangulations of the computational domain in its non-symmetric formulation

$$
\left(\begin{array}{cc}
\boldsymbol{A}_{\omega} & \boldsymbol{B} \\
-\boldsymbol{B}^{T} & \boldsymbol{C}
\end{array}\right)\left(\begin{array}{c}
\boldsymbol{u} \\
\boldsymbol{\Phi}
\end{array}\right)=\left(\begin{array}{c}
\boldsymbol{f} \\
-\boldsymbol{g}
\end{array}\right)
$$

where $\boldsymbol{A}_{\omega}:=\boldsymbol{A}-\omega^{2} \boldsymbol{I}$. We also consider the iterative solution of the Schur complement system

$$
\begin{aligned}
\boldsymbol{S u} & =\left(\boldsymbol{A}_{\omega}+\boldsymbol{B} \boldsymbol{C}^{-1} \boldsymbol{B}^{*}\right) \boldsymbol{u}=\boldsymbol{F}, \\
\boldsymbol{C \Phi} & =\boldsymbol{C u}-\boldsymbol{g}
\end{aligned}
$$


by the $\mathrm{CG}$ method with and without preconditioning (SC-CG/SC-PCG).

We first consider the results of a $2 \mathrm{D}$ simulation where $\Omega=(-1,+1)^{2}$ and the boundaries $\Gamma_{u}, \Gamma_{\sigma}, \Gamma_{\Phi}$ and $\Gamma_{D}$ are given as follows

$$
\begin{aligned}
& \Gamma_{u}:=[-1,+1] \times\{-1\} \cup\{+1\} \times[-1,+1], \\
& \Gamma_{\sigma}:=(-1,+1) \times\{+1\} \cup\{-1\} \times(-1,+1), \\
& \Gamma_{\Phi}:=[-1,+1] \times\{-1\} \cup\{-1\} \times[-1,+1], \\
& \Gamma_{D}:=(-1,+1) \times\{+1\} \cup\{+1\} \times(-1,+1) .
\end{aligned}
$$

The stopping criterion for the iterations has been chosen such that the residual gets smaller than $10^{-6}$. We list the number of iterations for SC-CG, GMRES and BICGSTAB with and without preconditioner. Since the effort for one iteration step is not directly comparable (there is an inner iteration in the application of the Schur complement), we have also measured the CPU-time (in seconds). Table 7.1 and Table 7.1 contain the results without and with the BPX-type preconditioner:

Table 1. Number of iterations and CPU-time (in seconds) for SC-CG and BICGSTAB/GMRES without preconditioner

\begin{tabular}{|c|c|c|c|c|c|c|}
\hline Level & \multicolumn{2}{|c|}{ SC-CG } & \multicolumn{2}{c|}{ BICGSTAB } & \multicolumn{2}{c|}{ GMRES } \\
\hline & time & iter & time & iter & time & iter \\
\hline \hline 3 & 0.15 & 74 & 0.10 & 65 & 0.14 & 17 \\
4 & 1.4 & 148 & 0.75 & 137 & 1.7 & 56 \\
5 & 29 & 311 & 7.6 & 324 & 32 & 206 \\
6 & 440 & 872 & 75 & 678 & 530 & 758 \\
\hline
\end{tabular}

Table 2. Number of iterations and CPU-time (in seconds) for SC-PCG and BICGSTAB/GMRES with preconditioner

\begin{tabular}{|c|c|c|c|c|c|c|}
\hline Level & \multicolumn{2}{|c|}{ SC-PCG } & \multicolumn{2}{c|}{ PBICGSTAB } & \multicolumn{2}{c|}{ PGMRES } \\
\hline & time & iter & time & iter & time & iter \\
\hline \hline 5 & 2.5 & 48 & 1.1 & 33 & 1.2 & 6 \\
6 & 12 & 52 & 5.2 & 39 & 5.9 & 7 \\
7 & 70 & 55 & 23 & 41 & 25 & 7 \\
8 & 290 & 57 & 92 & 44 & 100 & 8 \\
\hline
\end{tabular}

Using no preconditioning, BICGSTAB has clearly outperformed SC-CG and GMRES in terms of CPU-time and iterations. Applying the BPX-type preconditioner, PBICGSTAB and PGMRES perform similarly and both outclass SC-PCG. We have also performed 3D simulations for the computational domain $\Omega=(-1,+1)^{3}$ and the boundaries $\Gamma_{u}, \Gamma_{\sigma}, \Gamma_{\Phi}$, and $\Gamma_{D}$ specified according to

$$
\begin{aligned}
& \Gamma_{u}:=[-1,+1]^{2} \times\{-1\} \quad, \quad \Gamma_{\sigma}:=\partial \Omega \backslash \Gamma_{u}, \\
& \Gamma_{\Phi}:=\{-1\} \times[-1,+1]^{2}, \quad \Gamma_{D}:=\partial \Omega \backslash \Gamma_{\Phi} .
\end{aligned}
$$

Using the same termination criterion, the performance of SC-CG, BI-CGSTAB and GMRES with and without preconditioning was roughly the same as in the $2 \mathrm{D}$ case.
In order to illustrate the dependence of SC-CG and SC-PCG on the refinement level, Figure 9 displays the number of iterations as a function of the refinement level $\ell$ for SC-CG (blue line) and SC-PCG with the BPX preconditioner (red line). For comparison, the results for SC-PCG with the hierarchical type preconditioner (cf., e.g., $[41,59]$ ) are shown as well (green line). The results clearly show the exponential growth of the condition number of SC-PCG in $\ell$ and the level independence of SC-PCG with the BPX preconditioner at least in 2D (in the $3 \mathrm{D}$ case the asymptotics has not yet been reached). Likewise, the theoretically predicted growth of the condition number like $O\left((\ell+1)^{2}\right)$ for $d=2$ and like $O\left(2^{\ell}\right)$ for $d=3$ of SC-PCG with the hierarchical type preconditioner is reflected by the numerical results as well.
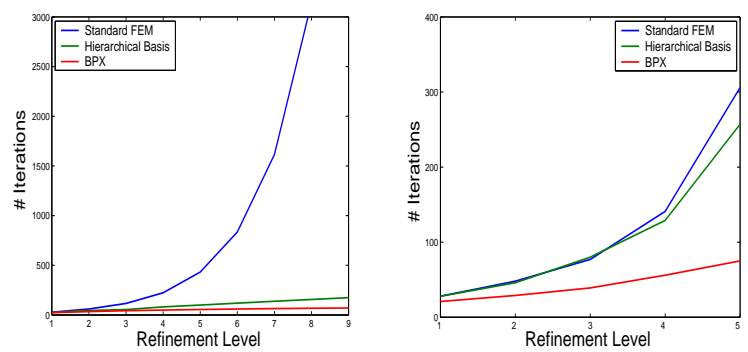

Fig. 9. Number of iterations required to reach a fixed tolerance $T O L=10^{-6} ; 2 \mathrm{D}$ simulation (left) and 3D simulation (right)

\subsection{Surface Acoustic Wave Device Simulation}

The piezoelectric material used for the SAW chip in our calculations is lithium niobate $\left(\mathrm{LiNbO}_{3}\right)$. Usually, one is interested in very large monocrystals appearing only randomly in nature. However, sophisticated production procedures for all technologically relevant materials are at hand. Depending on the cut used for the special device the monocrystals are sawed. For details concerning production procedures, natural appearances and the material constants stated here we refer to [61] and the references therein.

The material moduli are given here in a way such that the coordinate $x_{3}$-axis is identical with the polar axis $Z$ along which rotatory polarization occurs (the crystallographic $Z$-axis). By convention, the crystallographic axes are denoted by $X, Y, Z$, while the coordinate axes are denoted $x_{1}, x_{2}, x_{3}$.

Lithium niobate is an extremely versatile crystal material. It possesses a very high Curie temperature and excellent piezoelectric coupling coefficient making it attractive for ultrasonic device applications. Lithium niobate possesses a number of useful cuts that are extensively used in transducer applications, e.g. $Y Z \mathrm{LiNbO}_{3}$ (i.e. $Y$-axis crystal cut, $Z$-axis propagation) or $128^{\circ}$ rotated $Y X \mathrm{LiNbO}_{3}$. Material moduli are given for room temperature $\left(20^{\circ} \mathrm{C}\right)$ in the following table: 
Table 3. Material Moduli for $128^{\circ}$ rotated $Y X \mathrm{LiNbO}_{3}$ (note that $c_{11}=c_{22}, c_{13}=c_{23}, c_{14}=-c_{24}=c_{56}, c_{44}=c_{55}$ and $\left.e_{22}=-e_{16}\right)$

\begin{tabular}{|c|c|c|c|c|c|c|c|}
\hline $\begin{array}{c}c \\
{\left[10^{10} \frac{\mathrm{N}}{\mathrm{m}^{2}}\right]}\end{array}$ & $\begin{array}{c}c_{11} \\
20.3\end{array}$ & $\begin{array}{l}c_{12} \\
5.3\end{array}$ & $\begin{array}{l}c_{13} \\
7.5\end{array}$ & $\begin{array}{l}c_{14} \\
0.9\end{array}$ & $\begin{array}{c}c_{33} \\
24.5\end{array}$ & $\begin{array}{l}c_{44} \\
6.0\end{array}$ & $\begin{array}{l}c_{66} \\
7.5\end{array}$ \\
\hline $\begin{array}{c}e \\
{\left[\frac{C}{m^{2}}\right]}\end{array}$ & $\begin{array}{r}e_{15} \\
\quad 3\end{array}$ & $e_{24}$ & $e_{22}$ & $\begin{array}{l}-e_{21} \\
5\end{array}$ & $\begin{array}{r}e_{31}= \\
0\end{array}$ & & $\begin{array}{l}e_{33} \\
1.3\end{array}$ \\
\hline$\left[\begin{array}{c}\boldsymbol{\epsilon} \\
{\left[10^{-12} \frac{\boldsymbol{F}}{\boldsymbol{m}}\right]}\end{array}\right.$ & \multicolumn{4}{|c|}{$\begin{array}{c}\epsilon_{11}=\epsilon_{22} \\
749.0\end{array}$} & \multicolumn{3}{|c|}{$\begin{array}{c}\epsilon_{33} \\
253.2\end{array}$} \\
\hline
\end{tabular}

The constants given here are for crystal geometries coinciding with the coordinate planes. For some technological reasons, different cuts of crystals are preferred in practice, i.e. a coordinate transformation is realized by

$$
\overline{\boldsymbol{x}}=\overline{\boldsymbol{x}}(\boldsymbol{x}),
$$

The material moduli in the new coordinate system are then regained by the tensor transformations

$$
\begin{gathered}
\bar{c}_{\bar{i} \bar{j} \bar{k} \bar{l}}=c_{i j k l} \frac{\partial \bar{x}_{\bar{i}}}{\partial x_{i}} \frac{\partial x_{j}}{\partial x_{\bar{j}}} \frac{\partial \bar{x}_{\bar{k}}}{\partial x_{k}} \frac{\partial x_{l}}{\partial x_{\bar{l}}}, \\
\bar{e}_{\bar{i} \bar{j} \bar{k}}=e_{i j k} \frac{\partial \bar{x}_{\bar{i}}}{\partial x_{i}} \frac{\partial \bar{x}_{\bar{j}}}{\partial x_{j}} \frac{\partial x_{k}}{\partial x_{\bar{k}}}, \\
\bar{\epsilon}_{\bar{i} \bar{j}}=\epsilon_{i j} \frac{\partial \bar{x}_{\bar{i}}}{\partial x_{i}} \frac{\partial x_{j}}{\partial x_{\bar{j}}} .
\end{gathered}
$$

Usually, a simple rigid rotation is undertaken, i.e. the coordinate transformation is linear, $\overline{\boldsymbol{x}}=\boldsymbol{T} \boldsymbol{x}$, and $\frac{\partial \bar{x}_{\bar{i}}}{\partial x_{i}}=T_{\bar{i} i}$ represent the direction cosines between the two frames of reference.

In this setting, the relationship between the crystallographic fundamental orthogonal system of axes $X, Y, Z$ and the coordinate axes $x_{1}, x_{2}, x_{3}$ must be known. Note, that there are piezoelectric materials where the orientations of the crystallographic unit cell axes do not align with the fundamental coordinate system, but usually constants are given for the fundamental coordinate system and we will not consider such materials anyway.

In transducer design, there is a simple standardized [27] way to provide this information: Here, the first two letters (out of $X, Y, Z$ ) denote the initial plate orientation, the first indicating the plate thickness, the second the plate length before any rotations. The remaining three symbols ( $t=$ thickness, $w=$ width, $l=$ length) are used to indicate the plate edges used for rotation, followed by a list of corresponding angles (see Figure 10 (left) for a $Y Z$-plate and Figure 10 (right) for a rotated $Y Z w-\phi$ plate).

For the surface acoustic wave device, we have used a reduced model in the $\left(x_{1}, x_{3}\right)$-plane assuming that all variables do not depend on $x_{2}$ and have no impact in the $x_{2}$-direction. The piezoelectric material is lithium niobate $\left(\mathrm{LiNbO}_{3}\right)$ with density $\rho=4630 \frac{\mathrm{kg}}{\mathrm{m}^{3}}$. This chip is operated at room temperature $\left(20^{\circ} \mathrm{C}\right)$. We remark that

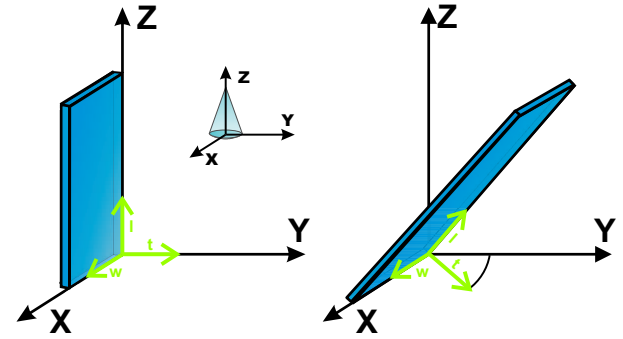

Fig. 10. Piezoelectric plate in crystallographic $X, Y, Z$ coordinate system; $Y Z$ orientation (left) and $Y Z w-\phi$ orientation (right)

the SAW devices can be cooled efficiently. Hence, the assumption of a constant operating temperature is justified. The used crystal cut is $Y X l 128^{\circ} \mathrm{LiNbO}_{3}$. The length $\ell$ and the height $h$ of the SAW chip have been chosen according to $\ell=1.2 \mathrm{~mm}$ and $h=0.6 \mathrm{~mm}$ so that $\Omega=(0,1.2) \times(0,0.6)$. The Dirichlet and Neumann boundary conditions have been specified according to

$$
\begin{gathered}
\boldsymbol{u}_{\Gamma}(\boldsymbol{x}, t)=\mathbf{0} \text { on } \Gamma_{u}, \quad \boldsymbol{\sigma}_{\boldsymbol{n}}(\boldsymbol{x}, t)=\mathbf{0} \text { on } \Gamma_{\sigma}, \\
\Phi_{\Gamma}(\boldsymbol{x}, t)=\hat{\Phi} \sin \left(\frac{2 \pi}{\lambda_{I D T}}\right) \sin (\omega t) \text { on } \Gamma_{\Phi}^{(1)}, \\
\Phi_{\Gamma}(\boldsymbol{x}, t)=0 \text { on } \Gamma_{\Phi}^{(2)}, \quad D_{\boldsymbol{n}}(\boldsymbol{x}, t)=0 \text { on } \Gamma_{D},
\end{gathered}
$$

where the Dirichlet boundaries are $\Gamma_{u}:=[0,1.2] \times\{0\}$, $\Gamma_{\Phi}^{(1)}:=[0.2,0.4] \times\{1.2\}, \Gamma_{\Phi}^{(2)}:=[0,1.2] \times\{0\}$, whereas $\Gamma_{\sigma}:=\partial \Omega \backslash \Gamma_{u}$ and $\Gamma_{D}=\partial \Omega \backslash\left(\Gamma_{\Phi}^{(1)} \cup \Gamma_{\Phi}^{(2)}\right)$ stand for the Neumann boundaries.

Concerning the wavelength $\lambda_{I D T}$ of the interdigital transducer and its operating frequency $f$, we have made the realistic choice $\lambda_{I D T}=40 \mu m$ and $f=\frac{\omega}{2 \pi}=$ $100 \mathrm{MHz}$.

We have discretized the computational domain by a nested hierarchy of simplicial triangulations of the computational domain $\Omega$ generated by uniform refinement of a given coarse mesh. It is well-known that for timeharmonic waves with increasing angular frequency $\omega=$ $\frac{2 \pi f}{\lambda}$ the finite element error grows, even if we account for a condition on the meshsize like $h \lesssim \lambda$. A common choice is $h \lesssim \frac{\lambda}{2}$ (i.e. two elements per wavelength), an estimate that guarantees an interpolation property for the used finite element spaces. However, an intrinsic analysis shows that an additional condition like $h \lesssim \sqrt{\lambda^{3}}$ is needed, if we want to control the finite element error (cf. [28]). Therefore, we have chosen the meshsize for the coarsest grid of the nested hierarchy accordingly.

Figures 11-13 show the amplitudes of the electric potential and the polarized Rayleigh waves, respectively. The amplitudes of the displacement waves are, as expected, in the region of nano-meters. The SAWs are strictly confined to the surface of the substrate. Their penetration depth into the piezoelectric material is in the range of one wavelength.

One of the most outstanding properties of surface acoustic wave propagation on piezoelectric materials is that the velocity of the SAW is independent of the applied frequency. In the case of $Y X l 128^{\circ} \mathrm{LiNbO}_{3}$ the 


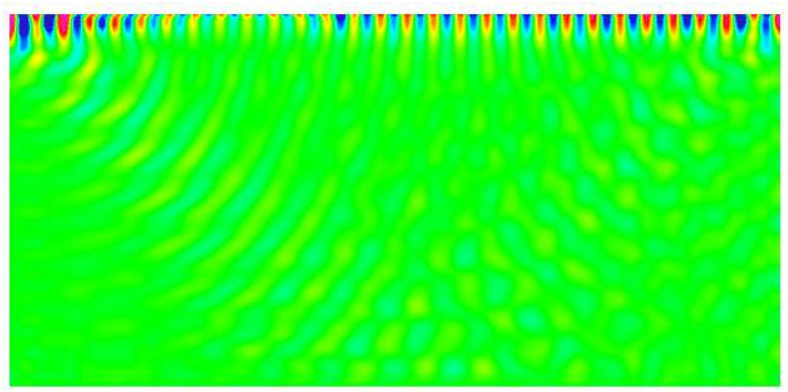

Fig. 11. Electric potential wave

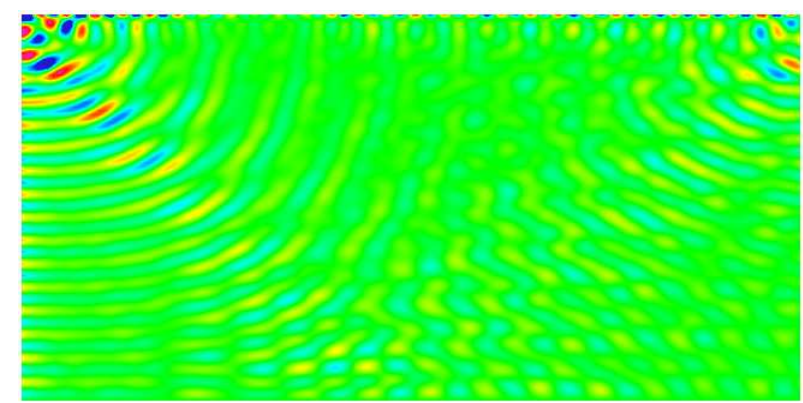

Fig. 12. Displacement wave amplitudes in $x_{1}$-direction

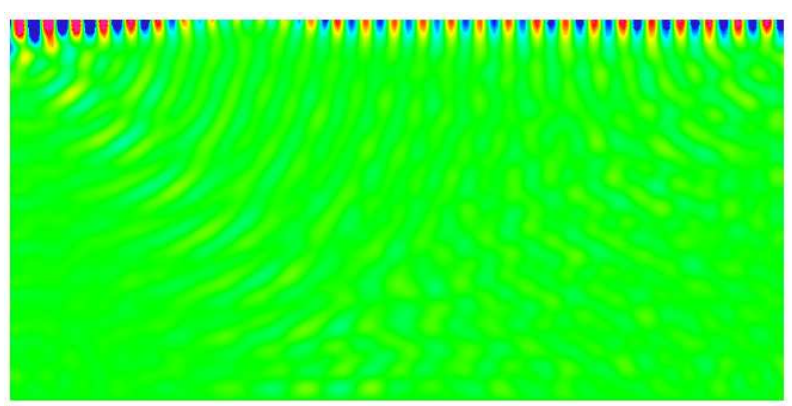

Fig. 13. Displacement wave amplitudes in $x_{2}$-direction

SAW velocity is given by $v=3992 \frac{\mathrm{m}}{\mathrm{s}}$, cf. [13]. Thus, for an excitation at the frequency $f=100 \mathrm{MHz}$ the theoretical wavelength of the SAW is given as $\lambda=\frac{v}{f} \approx 40 \mu \mathrm{m}$. Our calculations show the same wavelength for the SAW. Figure 14 also illustrates the piezoelectric wave for $f=$ $50 \mathrm{MHz}$. The wavelength of the SAW for $f=100 \mathrm{MHz}$ is half of that for $f=50 \mathrm{MHz}$.

We remark that the wavelength of an occurring electro-magnetical wave is in the region of approximately 0.3 $\mathrm{m}$. Hence, the negligence of this electro-magnetic wave in the modeling of piezoelectric SAW devices is justified.

The excitation of an IDT on the surface of a piezoelectric material leads to the generation of bulk acoustic waves (BAWs) as well as surface acoustic waves. These

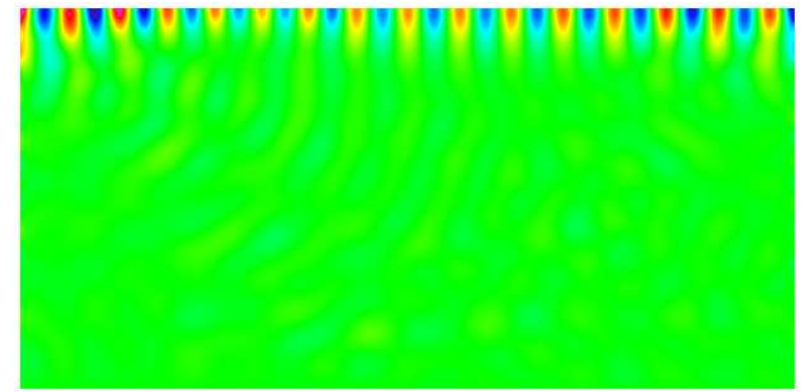

Fig. 14. Electric potential wave for $f=50 \mathrm{MHz}$

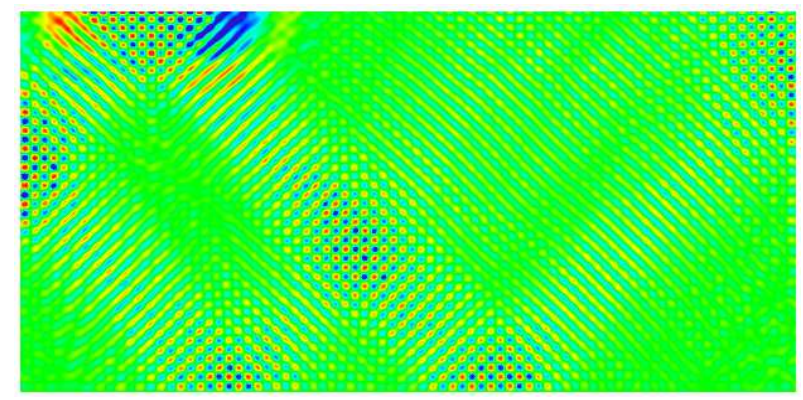

Fig. 15. Bulkwave Excitation

bulk waves can also be observed in our simulations in Figures 11-14. Technologically, they are desirably employed in solid-state circuits [13]. We refer to $[16,19$, $23,24,52]$ for finite element approximations of surface acoustic wave propagation in signal processing. However, for the SAW devices under consideration the presence of BAWs is unwanted, since the interference of BAWs with SAWs can lead to a complete loss of functionality of the device. Our approach is sufficiently general to simulate every kind of piezoelectric resonator. In Figure 15 we have used an $Y X l 38^{\circ}$ cut of $\mathrm{LiNbO}_{3}$ to generate a strong bulk acoustic wave at frequency $f=200 \mathrm{MHz}$.

We recall from Chapter 3 that Rayleigh surface waves characteristically show an elliptical displacement, i.e. the displacements in the $x_{1}$ - and $x_{2}$-direction are $90^{\circ}$ out of phase with one another. Additionally, the amplitude of the surface displacement in the $x_{2}$-direction is larger than that along the SAW propagation axis $x_{1}$. These observations are also true in our numerical computations, see Figures 16 and 17. In Figure 16, the displacements in the $x_{1}$ - and $x_{2}$-direction for a certain surface area are depicted. The $x_{2}$-displacements are flipped vertically for easier comparability. In Figure 17 a certain surface area is magnified and the vectors indicate the surface displacements. All numerical calculations show relatively strong reflections from the boundaries of the SAW device. In real devices these reflections are usually avoided by attaching some adhesive material to the side boundaries. An easy way to model such a damping is to introduce an additional term (the so-called gyroscopic term) 


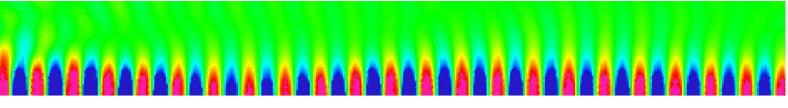

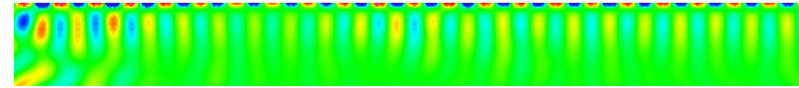

Fig. 16. Phaseshift of $x_{1^{-}}$and $x_{2^{-}}$(flipped) displacements

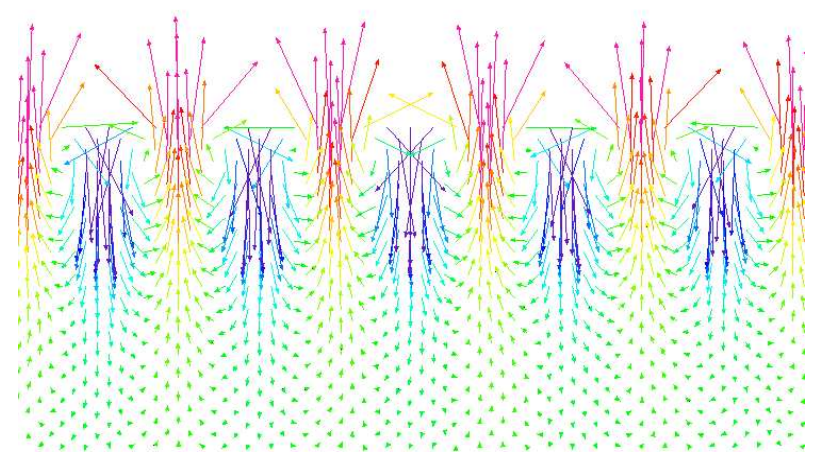

Fig. 17. Displacement vectors for the SAW

into the piezoelectric equations which now become

$$
\begin{gathered}
\rho \frac{\partial^{2} u_{i}}{\partial t^{2}}-\frac{\partial \beta_{i}}{\partial x_{j}} \frac{\partial^{3} u_{i}}{\partial^{2} x_{j} \partial t}- \\
-c_{i j k l} \frac{\partial^{2} u_{k}}{\partial x_{l} \partial x_{j}}-e_{k i j} \frac{\partial^{2} \Phi}{\partial x_{k} \partial x_{j}}=b_{i} \\
e_{i k l} \frac{\partial^{2} u_{k}}{\partial x_{l} \partial x_{i}}-\epsilon_{i j} \frac{\partial^{2} \Phi}{\partial x_{j} \partial x_{i}}=\beta_{i}
\end{gathered}
$$

cf. e.g. [6, 29]. Introducing such a damping at the boundaries of the bottom and left-hand side we indeed get less reflections and thus less disturbances for the SAW. This gets extraordinarily palpable, if we compare the $x_{1-}$ displacements in Figure 12 (calculations without damping) to the new calculations with damping term in Figure 18.

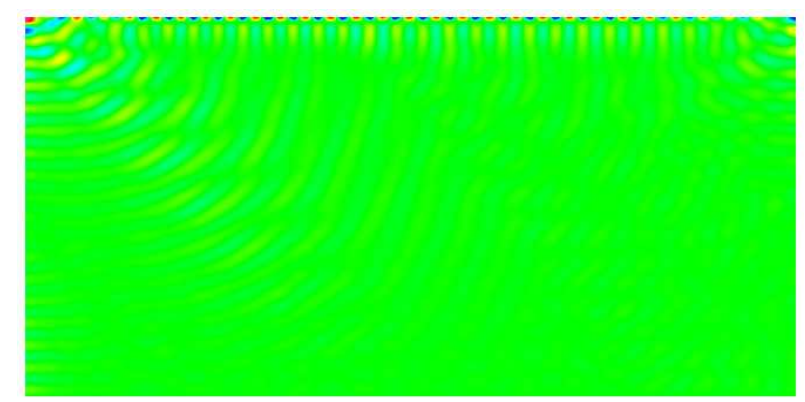

Fig. 18. $x_{1}$-displacements with damping

\section{References}

1. R.A. Adams; Sobolev spaces, Academic Press, New York - San Francisco - London, 1975

2. Advalytix AG, Personal communication, Brunnthal, 2005

3. B.A. Auld, Acoustic fields and waves in solids. Wiley, Chichester-New York, 1973

4. D.S. Ballantines (ed.), Acoustic Wave Sensors: Theory, Design and Physico-Chemical Applications, Academic Press, San Diego, 1997

5. R.E. Bank, B.D. Welfert, and H. Yserentant, A class of iterative methods for solving saddle point problems. Numer. Math., bf 56, 645-666, 1990

6. K.J. Bathe and E. Wilson, Numerical Methods in Finite Element Analysis, Prentice Hall, Englewood Cliffs, 1976

7. M. Benzi and G.H. Golub, A preconditioner for generalized saddle point problems. SIAM J. Matrix Anal. Appl., 26, 20-41, 2004

8. R. Bittner, M. Wassermeier, and A. Wixforth, Dynamical investigation of macromolecular hybridization bioassays, http://arxiv.org/pdf/physics/0207064, 2002

9. N. Botkin, M. Schlensog, M. Tewes, and V. Turova, A mathematical model of a biosensor, In: Proc. 4th Int. Conf. on Modeling and Simulation of Microsystems, Hilton Head Island, SC, USA, March 19-21, pp. 231-234, 2001

10. J.H. Bramble, J.E. Pasciak, and A.T. Vassilev, Analysis of the inexact Uzawa algorithm for saddle point problems. SIAM J. Numer. Anal., 34, 1072-1092, 1997

11. J.H. Bramble, J.E. Pasciak, and J. Xu, Parallel multilevel preconditioners. Math. Comp., 55, 1-22, 1990

12. F. Brezzi and M. Fortin, Mixed and Hybrid Finite Element Methods. Springer, Berlin-Heidelberg-New York, 1991

13. C. Campbell, Surface Acoustic Wave Devices and Their Signal Processing Applications. Academic Press, San Diego, 1989

14. Z.H. Cao, Fast Uzawa algorithm for generalized saddle point problems. Appl. Numer. Math., 46, 157-171, 2003

15. P. Ciarlet, Jr., J. Huang, and J. Zou, Some observations on generalized saddle-point problems. SIAM J. Matrix Anal. Appl., 25, 224-236, 2003

16. G. Endoh, K. Hashimoto, and M. Yamaguchi, Surface acoustic wave propagation characterization by finiteelement method and spectral domain analysis. Jpn. J. Appl. Phys. 34, 5B, 2632-2637, 1995

17. H.C. Elman and G.H. Golub, Inexact and preconditioned Uzawa algorithms for saddle point problems. SIAM J. Numer. Anal., 6, 1645-1661, 1994

18. A.C. Eringen and G.A. Maugin, Electrodynamics of Continua I. Foundations and Solid Media. Springer, Berlin-Heidelberg-New York, 1990

19. J. Fagerholm, Wave propagation in diffractive optical elements and surface-acoustic wave devices. CSC Research Report R07/96, Center for Scientific Computing, Helsinki University of Technology, Espoo, 1996

20. M. Feldmann and J. Hénaff. Surface Acoustic Waves for Signal Processing. Artech House, Boston, 1989

21. P. Grisvard, Elliptic Problems in Nonsmooth Domains. Pitman, Boston, 1985

22. K. Hasegawa and M. Koshiba, Finite-element solution of Rayleigh-wave scattering from reflective gratings on a piezoelectric substrate, IEEE Trans. Ultrason. Ferroelectr. Freq. Contr. 37, 99-105, 1990 
23. M. Hofer, M. Jungwirth, R. Lerch, and R. Weigel, Accurate and efficient modeling of SAW structures, Frequenz, 55, 64-72, 2001

24. M. Hofer, R. Lerch, N. Finger, G. Kovacs, J. Schöberl, and U. Langer, Finite element calculations of wave propagation and excitation in periodic piezoelectric systems, In: Proc. WCCM V, 5th World Congr. on Comput. Mech., Vienna, 2002

25. R.H.W. Hoppe and B. Wohlmuth, Adaptive multilevel techniques for mixed finite element discretizations of elliptic boundary value problems, SIAM J. Numer. Anal. 34, 1658-1681, 1997

26. R.H.W. Hoppe and B. Wohlmuth, Multilevel iterative solution and adaptive mesh refinement for mixed finite element discretizations, Appl. Numer. Math. 23, 97-117, 1997

27. Institute of Electrical and Electronics Engineers, Standard on piezoelectricity. IEEE Transactions on Sonics and Ultrasonics, 3, 1978

28. F. Ihlenburg, Finite Element Analysis of Acoustic Scattering. Springer, New York, 1998

29. H. Kardestuncer (ed.), Finite Element Handbook, McGraw-Hill, New York, 1987

30. G.S. Kino, Acoustic Waves: Devices, Imaging, and Analog Signal Processing. Prentice-Hall, Englewood Cliffs, 1987

31. A. Klawonn, Preconditioners for indefinite problems, Doctoral Dissertation, University of Münster, 1996

32. A. Klawonn, An optimal preconditioner for a class of saddle point problems with a penalty term. SIAM J. Sci. Comput., 19, 540-552, 1998

33. R. Lerch, Simulation of piezoelectric devices by two- and three-dimensional finite elements. IEEE Trans. Ultrasonics, Ferroelectrics and Frequency Control, 37, 233-247, 1990

34. M.F. Lewis, On Rayleigh waves and related propagating acoustic waves. In: Rayleigh-Wave Theory and Applications (E.A. Ash and E.G.S. Paigepages, eds.), pp. 37-58, Springer, Berlin-Heidelberg-New York, 1985

35. G.A. Maugin, Continuum Mechanics of Electromagnetic Solids. North-Holland, Amsterdam, 1987

36. D.P. Morgan, Surface-Wave Devices for Signal Processing. Elsevier, Amsterdam, 1991

37. J. Necas, Sur une méthode pour resoudre les équations aux dérivées partielles du type elliptique, voisine de la variationnelle. Ann. Sc. Norm. Super. Pisa, 16, 305-326, 1962

38. R.A. Nicolaides, Existence, uniqueness and approximation for generalized saddle point problems. SIAM J. Numer. Anal., 19, 349-357, 1982

39. J.A. Ogilvy, An approximate analysis of waves in layered piezoelectric plates from an interdigital source transducer. J. Phys. D, Applied Physics, 29, 876-884, 1996

40. A.A. Oliner, Acoustic Surface Waves, Springer, BerlinHeidelberg-New York, 1977

41. P. Oswald, Multilevel Finite Element Approximation: Theory and Applications. Teubner, Stuttgart, 1994

42. J. Pollard, B. Castrodale, Outlook for DNA microarrays: emerging applications and insights on optimizing microarray studies, Report. Cambridge Health Institute, Cambridge 2003

43. A. Rathgeber and A. Wixforth, Flat fluidics: Liquid handling without walls, http://arxiv.org/physics/0104079, 2001
44. Lord Rayleigh, On waves propagating along the plane surface of an elastic surface, Proc. London Math. Soc. 7, $4-11,1885$

45. C. Rocke, A.O. Govorov, A. Wixforth, G. Böhm, and G. Weimann, Exciton ionization by intense piezoelectric surface waves on a semiconductor quantum well, Phys. Rev. B 57, 6850, 1998

46. M. Rotter, C. Rocke, S. Böhm, A. Lorke, A. Wixforth, W. Rulle, and L. Korte, Single-chip fused hybrids for acousto-electric and acousto-optic applications, Appl. Phys. Lett. 70, 2097, 1997

47. A. Schmidt and K. G. Siebert, Design of Adaptive Finite Element Software: The Finite Element Toolbox ALBERTA, LNCSE, vol. 42, Springer, 2005.

48. A. Schmidt and K. G. Siebert, ALBERT - Software for scientific computations and applications, Acta Math. Univ. Comenianae, 70, 105-122, 2001.

49. D. Silvester and A. Wathen, Fast iterative solution of stabilised Stokes systems part I: Using simple diagonal preconditioners. SIAM J. Numer. Anal., 30, 630-649, 1993

50. D. Silvester and A. Wathen, Fast iterative solution of stabilised Stokes systems part II: Using general block preconditioners. SIAM J. Numer. Anal., 31, 1352-1367, 1994

51. M. Streibl, H.J. Kutschera, W. Sauer, and A. Wixforth, Numerical and experimental analysis of complex surface acoustic wave fields, In: Proc. IEEE Int. Ultrasonic Symp., San Juan, Puerto Rico, 2000 (D.E. Yuhas, ed.), 2001

52. P. Ventura, J.M. Hodé, J. Desbois, and M. Solal, Combined FEM and Green's function analysis of periodic SAW strucutre, application to the calculation of reflection and scattering parameters. IEEE Trans. Ultrasonics, Ferroelectrics and Frequency Control, 48, 1449-1461, 2001

53. R.M. White and F.W. Voltmer, Direct piezoelectric coupling to surface elastic waves. Appl. Phys. Lett., 17, 314316, 1965

54. A. Wixforth, Nano-Beben auf dem Chip: Akustische Oberflchenwellen als Photonen-Frderband. Physikalische Bltter, 54, 1998

55. A. Wixforth, C. Gauer, J. Scriba, M. Wassermeier, and R. Kirchner, Flat fluidics: A new route toward programmable biochips. booktitle In: Proc. of SPIE, 2003

56. A. Wixforth and J. Scriba, Nano-Pumpen fr das Chiplabor. GIT Laborfachzeitschrift, 5, 2002

57. A. Wixforth, J. Scriba, and G. Gauer, Flatland fluidics, mst news 5, 42-43, 2002

58. A. Wixforth, M. Wassermeier, J. Scriba, J.P. Kotthaus, G. Weimann, and W. Schlapp, Surface acoustic waves on GaAs.AlGaAs heterostructures, Phys. Rev. B 40, 7874, 1989

59. H. Yserentant, On the multi-level splitting of finite element spaces, Numer. Math. 49, 379-412, 1986

60. E. Zeidler, Nonlinear Functional Analysis and Its Applications. II/A: Linear Monotone Operators. Springer, Berlin-Heidelberg-New York, 1990

61. J. Zelenka, Piezoelectric Resonators and Their Applications. Elsevier, Amsterdam, 1986 